\title{
Langlands Duality for Finite-Dimensional Representations of Quantum Affine Algebras
}

\author{
To V. B. Matveev on his 65th birthday \\ EDWARD FRENKEL ${ }^{1}$ and DAVID HERNANDEZ ${ }^{2}$ \\ ${ }^{1}$ Department of Mathematics, University of California, Berkeley, CA 94720, USA. \\ e-mail: frenkel@math.berkeley.edu \\ ${ }^{2}$ Département de Mathématiques, Université Paris 7, 175 rue du Chevaleret, 75013 Paris, \\ France
}

Received: 15 November 2009 / Accepted: 6 September 2010

Published online: 22 September 2010 - (C) The Author(s) 2010. This article is published with open access at Springerlink.com

\begin{abstract}
We describe a correspondence (or duality) between the $q$-characters of finitedimensional representations of a quantum affine algebra and its Langlands dual in the spirit of Frenkel and Hernandez (Math Ann, to appear) and Frenkel and Reshetikhin (Commun Math Phys 197(1):1-32, 1998). We prove this duality for the Kirillov-Reshetikhin modules and their irreducible tensor products. In the course of the proof we introduce and construct "interpolating $(q, t)$-characters" depending on two parameters which interpolate between the $q$-characters of a quantum affine algebra and its Langlands dual.
\end{abstract}

Mathematics Subject Classification (2010). 17B37 (17B10, 81R50).

Keywords. Langlands duality, quantum affine algebras, Kirillov-Reshetikhin modules.

\section{Contents}

1 Introduction . . . . . . . . . . . . . . . . . . . . 217

2 Duality for the Ordinary Characters . . . . . . . . . . . . . . . . . . . 219

3 Double-Laced Cases . . . . . . . . . . . . . . . . . . . . . . . . 221

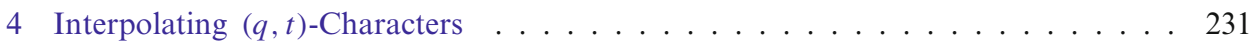

5 Triple-Laced Case . . . . . . . . . . . . . . . . . . . . 242

6 From Twisted to Untwisted Types . . . . . . . . . . . . . . . . . . . . . . 249

References . . . . . . . . . . . . . . . . . . . 260

\section{Introduction}

Let $\mathfrak{g}$ be a simple Lie algebra and $\widehat{\mathfrak{g}}$ the corresponding affine Kac-Moody algebra. In [7], Reshetikhin and one of the authors introduced a two-parameter deformed

E. Frenkel's work was supported in part by DARPA through the grant HR0011-09-1-0015 and by Fondation Sciences mathématiques de Paris.

D. Hernandez's work was supported partially by ANR through Project "Géométrie et Structures Algébriques Quantiques". 
$\mathcal{W}$-algebra $\mathcal{W}_{q, t}(\mathfrak{g})$. In the limit $t \rightarrow 1$ this deformed $\mathcal{W}$-algebra becomes commutative and gives rise to the Grothendieck ring of finite-dimensional representations of the quantum affine algebra $\mathcal{U}_{q}(\widehat{\mathfrak{g}})$. (The precise relation between the two is explained in [7] and [8].) On the other hand, in the limit when $q \rightarrow \epsilon$, where $\epsilon=1$ if $\mathfrak{g}$ is simply-laced and $\epsilon=\exp (\pi i / r), r$ being the lacing number of $\mathfrak{g}$, otherwise, this algebra contains a large center. It was conjectured in [7] that it gives rise to the Grothendieck ring of $U_{q}\left({ }^{L} \widehat{\mathfrak{g}}\right)$, where ${ }^{L} \widehat{\mathfrak{g}}$ is the Langlands dual Lie algebra of $\widehat{\mathfrak{g}}$. By definition, the Cartan matrix of ${ }^{L} \widehat{\mathfrak{g}}$ is the transpose of the Cartan matrix of $\widehat{\mathfrak{g}}$, so that ${ }^{L} \widehat{\mathfrak{g}}$ is a twisted affine algebra if $\mathfrak{g}$ is non-simply laced.

Thus, it appears that $\mathcal{W}_{q, t}(\mathfrak{g})$ interpolates between the Grothendieck rings of finite-dimensional representations of quantum affine algebras associated with $\widehat{\mathfrak{g}}$ and ${ }^{L} \widehat{\mathfrak{g}}$. This suggests that these representations should be related in some way. Examples of such a relation were given in [7], but general understanding of this phenomenon has been lacking. The goal of this paper is to elucidate and provide further evidence for this duality.

The finite-dimensional analogue of this duality has been studied in our previous paper [5], in which we have conjectured (and partially proved) the existence of a correspondence, or duality, between finite-dimensional representations of the quantum groups $U_{q}(\mathfrak{g})$ and $U_{q}\left({ }^{L} \mathfrak{g}\right) .{ }^{1}$ This duality may in fact be extended uniformly to integrable representations of quantized enveloping algebras associated with Kac-Moody algebras. But quantized enveloping algebras associated with the affine Kac-Moody algebras (quantum affine algebras for short) have another important class of representations; namely, the finite-dimensional representations. In this paper we describe a Langlands-type duality for these representations.

In this context the Langlands duality was first observed in $[7,8]$ using the so-called " $q$-characters" of finite-dimensional representations of quantum affine algebras. The theory of $q$-characters has been developed for untwisted quantum algebras in [8] and for twisted quantum affine algebras (which naturally appear in the Langlands dual situation) in [13]. The $q$-character of a simple representation characterizes its isomorphism class.

In this paper we conjecture a precise relation between the $q$-characters of finitedimensional representations of dual quantum affine algebras $U_{q}(\widehat{\mathfrak{g}})$ and $U_{q}\left({ }^{L} \widehat{\mathfrak{g}}\right)$. Namely, we conjecture that for any finite-dimensional representation $V$ of $U_{q}(\widehat{\mathfrak{g}})$ there exists an interpolating $(q, t)$-character, a polynomial which interpolates between the $q$-character of $V$ and the $t$-character of a certain representation of the Langlands dual algebra $U_{t}\left({ }^{L} \widehat{\mathfrak{g}}\right)$, which we call dual to $V$ (we discuss in which sense it is unique). Moreover, we prove this conjecture for an important class of representations, the Kirillov-Reshetikhin modules and their irreducible tensor products.

The $q$-characters are important in the study of integrable models of statistical mechanics (see, e.g., $[7,8]$ ), and therefore their duality indicates the existence of

${ }^{1}$ We have learned from McGerty that in the meantime he has been able to prove some of the conjectures of [5], see [19]. 
duality between the models associated with two Langlands dual affine Lie algebras. The existence of interpolating $(q, t)$-characters is closely related to [7, Conjecture 1], which also states the existence of interpolating expressions, but of a different kind. They are elements of a two-parameter non-commutative algebra (in fact, a Heisenberg algebra), whereas the interpolating characters that we introduce here are elements of a commutative algebra. It would be interesting to understand a precise relation between the two pictures.

We refer the reader to the Introduction of [5] for a discussion of a possible link between our results on the duality of finite-dimensional representations of $U_{q}(\mathfrak{g})$ and $U_{t}\left({ }^{L} \widehat{\mathfrak{g}}\right)$ and the geometric Langlands correspondence. This link was one of the motivations for the present paper.

Let us note that the technique and methods in the present paper are not generalizations of [5], but are new as we use the "rigidity" provided by the appearance of the spectral parameters in the context of quantum affine algebras. This allows us to construct the interpolating $(q, t)$-characters (which have a priori no clear analogues in finite types). Another difference is that instead of a projection from a weight lattice to the dual weight lattice, we introduce interpolating maps $\alpha(q, t), \beta(q, t)$ in the characters. These maps "kill" some of the terms when we specialize to the Langlands dual situation. Thus we obtain a much finer form of duality in the affine case than in the finite-dimensional case.

The paper is organized as follows: in Section 2 we recall the Langlands duality for quantum groups of finite type from [5]. Then we state consequences of the results of the present paper in terms of the ordinary characters (Theorem 2.3). In Section 3 we give a general conjecture about the duality at the level of $q$-characters. We state and start proving the main result of the present paper (Theorem 3.11) in the double-laced cases; namely, that the Kirillov-Reshetikhin modules and their irreducible tensor products satisfy the Langlands duality. The end of the proof uses results of Section 4 where interpolating $(q, t)$-characters are constructed in a systematic way (Theorem 4.4). The triple-laced is treated in Section 5 (Theorem 5.4 and Theorem 5.5) to complete the picture. In Section 6, we describe a reverse Langlands duality from twisted quantum affine algebras to untwisted quantum algebras and we prove analogous results for this duality (Theorems 6.8 and 6.9).

\section{Duality for the Ordinary Characters}

Although most of the results of the present paper involve $q$-characters, some consequences of our results may be stated purely in terms of the ordinary characters. We explain these results in this Section as well as some motivations and results from [5].

Let $\mathfrak{g}$ be a finite-dimensional simple Lie algebra and $U_{q}(\mathfrak{g})$ the corresponding quantum group (see, e.g., [3]). We denote $r=\max _{i \in I}\left(r_{i}\right)$, where $I$ is the set of vertices of the Dynkin diagram of $\mathfrak{g}$ and the $r_{i}$ are the corresponding labels. This is the lacing number of $\mathfrak{g}$ (note that it was denoted by $r^{\vee}$ in $[7,8]$ ). In some particular 
cases, we will not make the choice $\min _{i \in I}\left(r_{i}\right)=1$ (that is we multiply the standard labels by a coefficient).

The Cartan matrix of $\mathfrak{g}$ will be denoted by $C=\left(C_{i, j}\right)_{i, j \in I}$. By definition, the Langlands dual Lie algebra ${ }^{L} \mathfrak{g}$ has the Cartan matrix $C^{t}$, the transpose of the Cartan matrix $C$ of $\mathfrak{g}$.

Let

$$
P=\sum_{i \in I} \mathbb{Z} \omega_{i}
$$

be the weight lattice of $\mathfrak{g}$ and $P^{+} \subset P$ the set of dominant weights. For $i \in I$ let $r_{i}^{\vee}=1+r-r_{i}$ and consider the sublattice

$$
P^{\prime}=\sum_{i \in I} r_{i}^{\vee} \mathbb{Z} \omega_{i} \subset P
$$

Let

$$
P^{L}=\sum_{i \in I} \mathbb{Z} \check{\omega}_{i}
$$

be the weight lattice of ${ }^{L} \mathfrak{g}$. Consider the map $\Pi: P \rightarrow P^{L}$ defined by

$$
\Pi(\lambda)=\sum_{i \in I} \lambda\left(\check{\alpha}_{i}\right)\left(r_{i}^{\vee}\right)^{-1} \breve{\omega}_{i}
$$

if $\lambda \in P^{\prime}$ and $\Pi(\lambda)=0$, otherwise. Clearly, $\Pi$ is surjective.

Let Rep $\mathfrak{g}$ be the Grothendieck ring of finite-dimensional representations of $\mathfrak{g}$. We have the character homomorphism

$$
\chi: \operatorname{Rep} \mathfrak{g} \rightarrow \mathbb{Z}[P]=\mathbb{Z}\left[y_{i}^{ \pm 1}\right],
$$

where $y_{i}=e^{\omega_{i}}$. It sends an irreducible representation $L(\lambda)$ of $\mathfrak{g}$ with highest weight $\lambda \in P^{+}$to its character, which we will denote by $\chi(\lambda)$. We denote the character homomorphism for ${ }^{L} \mathfrak{g}$ by $\chi^{L}$. We use the obvious partial ordering $\preceq$ on polynomials. It was proved in [5] that for any $\lambda \in P^{+}, \Pi(\chi(\lambda))$ is in the image of $\chi^{L}$. Moreover, we have the following:

THEOREM 2.1. [5] For any $\lambda \in P^{+}, \Pi(\chi(\lambda)) \succeq \chi^{L}(\Pi(\lambda))$.

Let $q, t \in \mathbb{C}^{\times}$be such that $q^{\mathbb{Z}} \cap t^{\mathbb{Z}}=\{1\}$. Let $U_{q}(\widehat{\mathfrak{g}})$ be an untwisted quantum affine algebra which is not Langlands self-dual. Let $V$ be a simple finite-dimensional representation of $U_{q}(\widehat{\mathfrak{g}})$ which is of highest weight $\lambda$ in $P^{\prime}$ when viewed as a $U_{q}(\mathfrak{g})$-module. We conjecture the following:

CONJECTURE 2.2. There exists an irreducible representation $V^{L}$ of $U_{t}\left(\widehat{\mathfrak{g}}^{L}\right)$ of highest weight $\Pi(\lambda)$ such that $\Pi(\chi(V)) \succeq \chi^{L}\left(V^{L}\right)$. 
Note that the Langlands dual representation $V^{L}$ is not necessarily unique. Uniqueness statements will be discussed later in a more precise form of Langlands duality.

As a consequence of the results of the present paper, we will prove the following:

THEOREM 2.3. The statement of Conjecture 2.2 is satisfied for any KirillovReshetikhin module $V$ over $U_{q}(\widehat{\mathfrak{g}})$, with the Langlands dual representation $V^{L}$ a Kirillov-Reshetikhin module over $U_{t}\left({ }^{L} \widehat{\mathfrak{g}}\right)$.

Note also that in contrast to [5], we use $t$ and not $-t$ for the quantization parameter of the Langlands dual quantum algebra. This is just a consequence of a different choice of normalization made in the present paper.

The following conjecture of [5] has been proved by McGerty in [19]: for any $\lambda \in$ $P^{+}, \Pi(\chi(\lambda))$ is the character of an actual (not only virtual) representation of ${ }^{L} \mathfrak{g}$. Therefore it is natural to make the following:

CONJECTURE 2.4. $\Pi(\chi(L))$ is the character of a representation of $U_{t}\left({ }^{L} \widehat{\mathfrak{g}}\right)$.

Again, this representation of $U_{t}\left({ }^{L} \widehat{\mathfrak{g}}\right)$ is not unique, but it is unique as a $U_{t}\left({ }^{L} \mathfrak{g}\right)$ module. However, it is not necessarily simple as a $U_{t}\left({ }^{L} \mathfrak{g}\right)$-module. As an example, for a 5-dimensional fundamental representation of $U_{q}\left(C_{2}^{(1)}\right)$, the Langlands dual representation decomposes into a sum of a 4-dimensional fundamental representation and the trivial representation of $U_{t}\left(A_{3}^{(2)}\right)$ (see the corresponding $q$-characters in Section 3.5).

\section{Double-Laced Cases}

In this section we suppose that the lacing number $r$ is equal to 2 (the case $r=$ 3 will be treated in Section 5). We will exclude from consideration the Langlands self-dual quantum affine Lie algebras (affinizations of simply-laced ones and those $\left.A_{2 n}^{(2)}\right)$.

We have $I=I_{1} \sqcup I_{2}$ where $I_{k}=\left\{i \in I \mid r_{i}=k\right\}$. For $i, j \in I$, we denote $i \sim j$ if $C_{i, j}<$ 0 . We can choose $\phi: I \rightarrow\{1,0\}$ such that $i \sim j \Rightarrow \phi(i)+\phi(j)=1$ and $C_{i, j}=-2 \Rightarrow$ $\phi(i)=1$.

\subsection{REMINDER ON $q$-CHARACTERS AND THEIR TWISTED ANALOGUES}

We recall the notion of $q$-characters first introduced in [8] for untwisted quantum affine algebras (see [2] for a recent survey) and generalized in [13] to the twisted cases.

The $q$-character homomorphism [8] is an injective ring morphism

$$
\chi_{q}: \operatorname{Rep}\left(U_{q}(\widehat{\mathfrak{g}})\right) \rightarrow \mathcal{Y}_{q}=\mathbb{Z}\left[Y_{i, a}^{ \pm 1}\right]_{i \in I, a \in q^{\mathbb{Z}}}
$$


(without loss of generality, we restrict ourselves to the tensor subcategory of finitedimensional representations whose $q$-characters are in $\mathcal{Y}_{q}$ ). By removing the spectral parameter $a$, that is to say by replacing each $Y_{i, a}$ by $y_{i}$, we recover the usual character map for the $U_{q}(\mathfrak{g})$-module obtained by restriction of $U_{q}(\widehat{\mathfrak{g}})$-module. In particular, each monomial has a weight which is an element of $P$. For $i \in I$, let $q_{i}=q^{r_{i}}$.

THEOREM 3.1. [6] We have

$$
\operatorname{Im}\left(\chi_{q}\right)=\bigcap_{i \in I} \mathfrak{K}_{i, q},
$$

where $\mathfrak{K}_{i, q}=\mathbb{Z}\left[Y_{j, a}^{ \pm 1}, Y_{i, a}\left(1+A_{i, a q_{i}}^{-1}\right)\right]_{j \neq i, a \in q^{\mathbb{Z}}}$ and

$$
A_{i, a}=Y_{i, a q_{i}^{-1}} Y_{i, a q_{i}} \times \prod_{j \in I, C_{j, i}=-1} Y_{j, a}^{-1} \times \prod_{j \in I, C_{j, i}=-2} Y_{j, a q^{-1}}^{-1} Y_{j, a q}^{-1} .
$$

A monomial in $\mathcal{Y}_{q}$ is called dominant if it is a product of positive powers of the $Y_{i, a}$ (for $i \in I, a \in q^{\mathbb{Z}}$ ). A simple $U_{q}(\widehat{\mathfrak{g}})$-module is characterized by the highest monomial (in the sense of its weight) of its $q$-character (this is equivalent to the data of the Drinfeld polynomials, see [3]). This monomial is dominant. Any element of $\operatorname{Im}\left(\chi_{q}\right)$ is characterized by the list of its dominant monomials. A $U_{q}(\widehat{\mathfrak{g}})$ module is said to be affine-minuscule if its $q$-character has a unique dominant monomial.

DEFINITION 3.2. A Kirillov-Reshetikhin (KR) module of $U_{q}(\widehat{\mathfrak{g}})$ is a simple module with the highest monomial of the form $Y_{i, a} Y_{i, a q_{i}^{2}} \cdots Y_{i, a q_{i}^{2(k-1)}}$.

We have the following result which is due to Nakajima [20,21] in the simplylaced case and [11] in general (note that for $k=1$ this was proved in [6] in the untwisted case):

THEOREM 3.3. The KR modules of $U_{q}(\widehat{\mathfrak{g}})$ are affine-minuscule.

Now let us look at the Langlands dual situation, i.e., finite-dimensional representations of the twisted quantum affine algebra $U_{t}\left({ }^{L} \widehat{\mathfrak{g}}\right)$. We set $\epsilon=e^{i \pi / 2}$ and $I_{1}^{\vee}=$ $I_{2}, I_{2}^{\vee}=I_{1}$.

The twisted $t$-character morphism [13] is an injective ring homomorphism (we work in a subcategory defined as in the untwisted case)

$$
\chi_{t}^{L}: \operatorname{Rep}\left({ }^{L} U_{t}(\widehat{\mathfrak{g}})\right) \rightarrow \mathcal{Y}_{t}^{L}=\mathbb{Z}\left[Z_{i, a^{r}}^{ \pm 1}\right]_{a \in \epsilon^{\mathbb{Z}} t^{\mathbb{Z}}, i \in I}
$$


THEOREM 3.4. [13] We have

$$
\operatorname{Im}\left(\chi_{t}^{L}\right)=\bigcap_{i \in I} \mathfrak{K}_{i, t}^{L},
$$

where

$$
\mathfrak{K}_{i, t}^{L}=\mathbb{Z}\left[Z_{j, a^{r_{j}^{\vee}}}^{ \pm 1}, Z_{i, a^{r_{i}^{\vee}}}\left(1+B_{i,(a t)^{r_{i}^{\vee}}}^{-1}\right]_{j \neq i, a \in \epsilon^{\mathbb{Z}} t^{\mathbb{Z}}}\right.
$$

and

$$
B_{i, a}= \begin{cases}Z_{i, a t^{2}} Z_{i, a t^{-2}} \times \prod_{j \sim i \mid j \in I_{2}^{\vee}} Z_{j, a}^{-1} \times \prod_{j \sim i \mid j \in I_{1}^{\vee}} \prod_{a^{\prime} \in \mathbb{Z}_{\mathbb{Z}} \mathbb{Z}_{\mid} \mid\left(a^{\prime}\right)^{2}=a} Z_{j, a^{\prime}}^{-1} & \text { if } i \in I_{2}^{\vee}, \\ Z_{i, a t} Z_{i, a t^{-1}} \times \prod_{j \sim i \mid j \in I_{2}^{\vee}} Z_{j, a^{2}}^{-1} \times \prod_{j \sim i \mid j \in I_{1}^{\vee}} Z_{j, a}^{-1} & \text { if } i \in I_{1}^{\vee} .\end{cases}
$$

Note that a special definition should be used [13] for the $B_{i, a}$ in the case of type $A_{2 n}^{(2)}$, but this case is not considered here.

We have the notions of dominant monomial, affine-minuscule module and KR module as in the untwisted case. Any element of $\operatorname{Im}\left(\chi_{t}^{L}\right)$ is again characterized by its dominant monomials and we have

THEOREM 3.5. [13] The KR modules of $U_{t}\left(L_{\widehat{\mathfrak{g}}}\right)$ are affine-minuscule.

\subsection{THE INTERPOLATING $(q, t)$-CHARACTER RING}

We first treat study the duality from untwisted quantum affine algebras to twisted quantum affine algebras. The reverse Langlands duality will be treated later.

We introduce the interpolating $(q, t)$-characters, which interpolate between $q$-characters of an untwisted quantum affine algebra and the twisted $t$-characters of its Langlands dual. To do it, we first need to define an interpolating ring for the target rings of $q$ - and $t$-character homomorphisms.

We also need the function $\alpha(q, t)$ such that $\alpha(q, 1)=1$ and $\alpha(\epsilon, t)=0$ defined in $[7,8]$ (see also [5] for an elementary natural way to introduce it in the framework of current algebras) by the formula

$$
\alpha(q, t)=\frac{\left(q+q^{-1}\right)\left(q t-q^{-1} t^{-1}\right)}{q^{2} t-q^{-2} t^{-1}} .
$$

Let $\mathcal{C}=q^{\mathbb{Z}} t^{\mathbb{Z}}$. Consider the ring

$$
\begin{aligned}
& \mathcal{Y}_{q, t}=\mathbb{Z}\left[W_{i, a}^{ \pm 1}, \alpha Y_{i, a}^{ \pm 1}, \alpha\right]_{i \in I, a \in \mathcal{C}} \subset \mathbb{Z}\left[Y_{i, a}^{ \pm 1}, \alpha\right]_{i \in I, a \in \mathcal{C},}, \\
& \text { where } W_{i, a}= \begin{cases}Y_{i, a} & \text { if } i \in I_{2}, \\
Y_{i, a q^{-1}} Y_{i, a q} & \text { if } i \in I_{1} .\end{cases}
\end{aligned}
$$


For $a \in \mathcal{C}$, we will use the following identification for $i \in I_{1}$ and $j \in I_{2}$ :

$$
Y_{i, a} Y_{i,-a}=Z_{i, a^{2}(-1)^{\phi(i)}} \quad \text { and } \quad Y_{j, a}=Z_{j, a(-1)^{\phi(i)}} .
$$

We then have surjective specialization maps, respectively, at $t=1$ and $q=\epsilon$,

$$
\begin{aligned}
& \Pi_{q}: \mathcal{Y}_{q, t} \rightarrow \mathbb{Z}\left[Y_{i, a}^{ \pm 1}\right]_{i \in I, a \in q^{\mathbb{Z}}}=\mathcal{Y}_{q}, \\
& \Pi_{t}: \mathcal{Y}_{q, t} \rightarrow \mathbb{Z}\left[Z_{i, a^{r_{i}}}^{ \pm 1}\right]_{i \in I, a \in \epsilon^{\mathbb{Z}} t}=\mathcal{Y}_{t}^{L} .
\end{aligned}
$$

We have the ideals

$$
\begin{aligned}
& \operatorname{Ker}\left(\Pi_{q}\right)=\left\langle(\alpha-1),\left(W_{i, a}-W_{i, a t}\right), \alpha\left(Y_{i, a}-Y_{i, a t}\right)\right\rangle_{i \in I, a \in \mathcal{C}}, \\
& \operatorname{Ker}\left(\Pi_{t}\right)=\left\langle\alpha,\left(W_{i, a q}-W_{i, a \epsilon}\right)\right\rangle_{i \in I, a \in \mathcal{C}} .
\end{aligned}
$$

Then we have the following:

LEMMA 3.6. The ideal $\operatorname{Ker}\left(\Pi_{q}\right) \cap \operatorname{Ker}\left(\Pi_{t}\right)$ is generated by the elements

$$
\alpha(\alpha-1), \alpha\left(Y_{i, a}-Y_{i, a t}\right),(\alpha-1)\left(W_{i, a q}-W_{i, a \epsilon}\right),\left(W_{i, a}-W_{i, a t}\right)\left(W_{j, b q}-W_{j, b \epsilon}\right),
$$

for $i, j \in I, a, b \in \mathcal{C}$.

Proof. First, the ideal $\mathfrak{I}$ generated by these elements is clearly included the intersection $\operatorname{Ker}\left(\Pi_{q}\right) \cap \operatorname{Ker}\left(\Pi_{t}\right)$ and so we work modulo $\mathfrak{I}$. We denote by $\equiv$ the equality modulo I. Now consider an element $\chi$ in the intersection. It is of the form

$$
\begin{aligned}
\chi & \equiv(\alpha-1) \chi(q, t)+\sum_{i \in I, l, r \in \mathbb{Z}}\left(W_{i, q^{l} t^{r}}-W_{i, q^{l} t^{r+1}}\right) \chi_{i, l, r}(q, t) \\
& \equiv(\alpha-1) \chi(\epsilon, t)+\sum_{i, l, r}\left(W_{i, q^{l} t^{r}}-W_{i, q^{l} t^{r+1}}\right) \chi_{i, l, r}(\epsilon, t)
\end{aligned}
$$

If we evaluate at $q=\epsilon$, we get

$$
\chi(\epsilon, t) \equiv \sum_{i, l, r}\left(W_{i, \epsilon^{l} t^{r}}-W_{\left.i, \epsilon^{l} t^{r+1}\right)} \chi_{i, l, r}(\epsilon, t),\right.
$$

and so

$$
\chi \equiv \sum_{i, l, r}\left(W_{i, q^{l} t^{r}}-W_{i, q^{l} t^{r+1}}+(\alpha-1)\left(W_{i, \epsilon^{l} t^{r}}-W_{i, \epsilon^{l} t^{r+1}}\right)\right) \chi_{i, l, r}(\epsilon, t)
$$

As $(\alpha-1)\left(W_{i, \epsilon^{l} t^{r}}-W_{i, \epsilon^{l} t^{r+1}}\right) \equiv-\left(W_{i, q^{l} t^{r}}-W_{i, q^{l} t^{r+1}}\right)$, we get

$$
\sum_{i, l, r}\left(W_{i, q^{l} t^{r}}-W_{i, q^{l} t^{r+1}}-\left(W_{i, q^{l} t^{r}}-W_{i, q^{l} t^{r+1}}\right)\right) \chi_{i, l, r}(\epsilon, t) \equiv 0 .
$$

This concludes the proof. 
We will work in the ring

$$
\widetilde{\mathcal{Y}}_{q, t}=\mathcal{Y}_{q, t} /\left(\operatorname{Ker}\left(\Pi_{q}\right) \cap \operatorname{Ker}\left(\Pi_{t}\right)\right) .
$$

Note that $\widetilde{\mathcal{Y}}_{q, t}$ has zero divisors as $\alpha^{2}=\alpha$ in $\widetilde{\mathcal{Y}}_{q, t}$.

By a monomial in $\widetilde{\mathcal{Y}}_{q, t}$ we will understand an element $m$ of the form $(\lambda+\mu \alpha) M$, where $\lambda, \mu \in \mathbb{Z}$ and $M$ is a monomial in the $Y_{j, a}^{ \pm 1}$. Note that a monomial may be written in various way as for example $\alpha Y_{i, a}=\alpha Y_{i, a t}$ and $(1-\alpha) Y_{i, a q^{4}}=(1-\alpha) Y_{i, a}$. A monomial is said to be $i$-dominant if it can be written by using only the $\alpha, Y_{i, a}$ and $Y_{j, a}^{ \pm 1}$ where $j \neq i$. Let $B_{i}$ be the set of $i$-dominant monomials and for $J \subset I$, let $B_{J}=\cap_{j \in J} B_{j}$. Finally, $B=B_{I}$ is the set of dominant monomials.

\subsection{SUBALGEBRAS OF $\widetilde{\mathcal{Y}}_{q, t}$}

DEFINITION 3.7. For $i \in I$ and $a \in \mathcal{C}$ we define

$$
\widetilde{A}_{i, a}=Y_{i, a\left(q_{i} t\right)^{-1}} Y_{i, a q_{i} t} \times \prod_{j \in I, C_{j, i}=-1} Y_{j, a}^{-1} \times \prod_{j \in I, C_{j, i}=-2} Y_{j, a q^{-1}}^{-1} Y_{j, a q}^{-1} .
$$

Note that the definition of $\widetilde{A}_{i, a}$ is not symmetric in $q, t$. For $i \in I_{2}, a \in \mathcal{C}$ we have $\widetilde{A}_{i, a}^{ \pm 1} \in \mathcal{Y}_{q, t}$, and for $i \in I_{1}$ we have $\alpha \widetilde{A}_{i, a}^{ \pm 1} \in \mathcal{Y}_{q, t}$ and $\left(\widetilde{A}_{i, a q^{-1}} \widetilde{A}_{i, a q}\right)^{ \pm 1} \in \mathcal{Y}_{q, t}$. But the specialization maps $\Pi_{q}, \Pi_{t}$ can be applied to any $\widetilde{A}_{i, a}$ and we have the following:

LEMMA 3.8. We have $\Pi_{q}\left(\widetilde{A}_{i, a}\right)=A_{i, \Pi_{q}(a)}$ for $i \in I, a \in \mathcal{C}$.

We have $\Pi_{t}\left(\left(\widetilde{A}_{i, a q^{-1}} \widetilde{A}_{i, a q}\right)\right)=B_{i,\left(\Pi_{t}(a)\right)^{2}(-1)^{\phi(i)}}$ for $i \in I_{1}, a \in \mathcal{C}$.

We have $\Pi_{t}\left(\widetilde{A}_{i, a}\right)=B_{i,-\Pi_{t}(a)(-1)^{\phi(i)}}$ for $i \in I_{2}, a \in \mathcal{C}$.

Proof. The first point is clear.

Let $a^{\prime}=\Pi_{t}(a)$. For $i \in I_{1}$, the specialization of $\widetilde{A}_{i, a q^{-1}} \widetilde{A}_{i, a q}$ at $q=\epsilon$ is

$$
\begin{aligned}
& \left(Y_{i,-a^{\prime} t^{-1}} Y_{i, a^{\prime} t^{-1}}\right)\left(Y_{i, a^{\prime} t} Y_{i,-a^{\prime} t}\right) \times \prod_{j \in I_{1}, j \sim i}\left(Y_{j, a^{\prime} \epsilon} Y_{j,-a^{\prime} \epsilon}\right)^{-1} \times \prod_{j \in I_{2}, j \sim i} Y_{j, a^{\prime} \epsilon}^{-1} Y_{j,-a^{\prime} \epsilon}^{-1} \\
& =Z_{i,\left(a^{\prime}\right)^{2} t^{-2}(-1)^{\phi(i)}} Z_{i,\left(a^{\prime}\right)^{2} t^{2}(-1)^{\phi(i)} \times} \prod_{j \in I_{1}, j \sim i} Z_{j,\left(a^{\prime}\right)^{2}(-1)^{\phi(i)}}^{-1} \times \prod_{j \in I, j \sim i} Z_{j, a^{\prime} \epsilon}^{-1} Z_{j,-a^{\prime} \epsilon}^{-1} .
\end{aligned}
$$

Note that if there is $j \in I_{2}$ such that $j \sim i$, by definition of $\phi$ we have $\phi(j)=0$ and $\phi(i)=1$. That is why there is no $\phi$ in the last factor of the product.

For $i \in I_{2}$, the specialization of $\widetilde{A}_{i, a}$ at $q=\epsilon$ is

$$
\begin{aligned}
& Y_{i,-a^{\prime} t^{-1}} Y_{i,-a^{\prime} t} \times \prod_{j \in I_{2}, j \sim i} Y_{j, a^{\prime}}^{-1} \times \prod_{j \in I_{1}, j \sim i}\left(Y_{j,-a^{\prime} \epsilon} Y_{j, a^{\prime} \epsilon}\right)^{-1} \\
& \quad=Z_{i,-a^{\prime} t^{-1}(-1)^{\phi(i)}} Z_{i,-a^{\prime} t(-1)^{\phi(i)}} \times \prod_{j \in I_{2}, j \sim i} Z_{j,-a^{\prime}(-1)^{\phi(i)}}^{-1} \times \prod_{j \in I_{1}, j \sim i} Z_{j,\left(a^{\prime}\right)^{2}}^{-1} .
\end{aligned}
$$


Note that if there is $j \in I_{1}$ such that $j \sim i$, by definition of $\phi$ we have $\phi(j)=1$ and $\phi(i)=0$. That is why there is no $\phi$ in the last factor of the product.

For $i \in I_{1}$ consider the subalgebra of $\mathcal{Y}_{q, t}$

$$
\mathfrak{K}_{i, q, t}=\mathbb{Z}\left[W_{i, a}\left(1+\alpha \widetilde{A}_{i, a q^{2} t}^{-1}+\widetilde{A}_{i, a q^{2} t}^{-1} \widetilde{A}_{i, a t}^{-1}\right), \alpha Y_{i, a}\left(1+\widetilde{A}_{i, a q t}^{-1}\right), W_{j, a}^{ \pm 1}, \alpha Y_{j, a}^{ \pm 1}, \alpha\right]_{a \in \mathcal{C}, j \neq i},
$$

and for $i \in I_{2}$

$$
\mathfrak{K}_{i, q, t}=\mathbb{Z}\left[Y_{i, a}\left(1+\widetilde{A}_{i, a q^{2} t}^{-1}\right), W_{j, a}^{ \pm 1}, \alpha Y_{j, a}^{ \pm 1}, \alpha\right]_{a \in \mathcal{C}, j \neq i}
$$

Then we have the following:

LEMMA 3.9. For $i \in I$, we have $\Pi_{q}\left(\mathfrak{K}_{i, q, t}\right)=\mathfrak{K}_{i, q}$ and $\Pi_{t}\left(\mathfrak{K}_{i, q, t}\right)=\mathfrak{K}_{i, t}^{L}$.

Proof. For $i \in I_{1}$, we have

$$
\begin{aligned}
& \Pi_{q}\left(\mathfrak{K}_{i, q, t}\right)=\mathbb{Z}\left[Y_{i, a}\left(1+A_{i, a q}^{-1}\right), Y_{j, a}^{ \pm 1}\right]_{a \in q^{\mathbb{Z}}, j \neq i}=\mathfrak{K}_{i, q},
\end{aligned}
$$

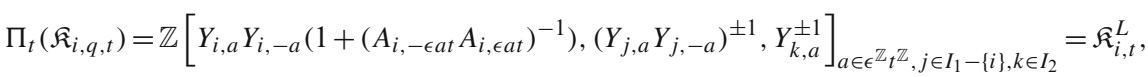

as by Lemma 3.8 we have

$$
Y_{i, a} Y_{i,-a}\left(1+\left(A_{i,-\epsilon a t} A_{i, \epsilon a t}\right)^{-1}\right)=Z_{i, a^{2}(-1)^{\phi(i)}}\left(1+B_{i, a^{2} t^{2}(-1)^{\phi(i)}}^{-1}\right) .
$$

For $i \in I_{2}$, we have

$$
\begin{aligned}
& \Pi_{q}\left(\mathfrak{K}_{i, q, t}\right)=\mathbb{Z}\left[Y_{i, a}\left(1+A_{i, a q^{2}}^{-1}\right), Y_{j, a}^{ \pm 1}\right]_{a \in q^{\mathbb{Z}}, j \neq i}=\mathfrak{K}_{i, q}, \\
& \Pi_{t}\left(\mathfrak{K}_{i, q, t}\right)=\mathbb{Z}\left[Y_{i, a}\left(1+A_{i,-a t}^{-1}\right),\left(Y_{j, a} Y_{j,-a}\right)^{ \pm 1}, Y_{k, a}^{ \pm 1}\right]_{a \in \epsilon^{\mathbb{Z}} t^{\mathbb{Z}}, j \in I_{1}, k \in I_{2}-\{i\}}=\mathfrak{K}_{i, t}^{L},
\end{aligned}
$$

as by Lemma 3.8 we have

$$
Y_{i, a}\left(1+A_{i,-a t}^{-1}\right)=Z_{i, a(-1)^{\phi(i)}}\left(1+B_{i, a(-1)^{\phi(i) t}}^{-1}\right) .
$$

We use the same notation $\mathfrak{K}_{i, q, t}$ for the image of the subalgebras $\mathfrak{K}_{i, q, t}$ in $\widetilde{\mathcal{Y}}_{q, t}$. For $J \subset I$ we define $\mathfrak{K}_{J}=\bigcap_{j \in J} \mathfrak{K}_{j}$ and we set $\mathfrak{K}=\mathfrak{K}_{I} \subset \tilde{\mathcal{Y}}_{q, t}$. 


\subsection{MAIN CONJECTURE AND MAIN THEOREM}

Let us define an analogue of $P^{\prime} \subset P$,

$$
\mathcal{Y}_{q}^{\prime}=\mathbb{Z}\left[Y_{i, a}^{ \pm 1}\right]_{i \in I_{2}, a \in q^{\mathbb{Z}}} \otimes \mathbb{Z}\left[\left(Y_{i, a q} Y_{i, a q^{-1}}\right)^{ \pm 1}\right]_{i \in I_{1}, a \in q^{\mathbb{Z}}} \subset \mathcal{Y}_{q} .
$$

We consider $\widehat{\Pi}: \mathcal{Y}_{q} \rightarrow \mathcal{Y}_{q}^{\prime}$ the projection on $\mathcal{Y}_{q}^{\prime}$ whose kernel is generated by monomials not in $\mathcal{Y}_{q}^{\prime}$.

Let $M \in \mathcal{Y}_{q}^{\prime}$ be a dominant monomial and $V$ the corresponding irreducible representation of $U_{q}(\widehat{\mathfrak{g}})$. A representation $V^{L}$ of $U_{t}\left({ }^{L} \widehat{\mathfrak{g}}\right)$ is said to be Langlands dual to $L(M)$ if there is a dominant monomial $\widetilde{M} \in \widetilde{\mathcal{Y}}_{q, t} \backslash \alpha \widetilde{\mathcal{Y}}_{q, t}$ and $\tilde{\chi}_{q} \in \mathfrak{K} \cap$ $\widetilde{M} \mathbb{Z}\left[\widetilde{A}_{i, a}^{-1}, \alpha\right]_{i \in I, a \in \mathcal{C}}$ such that

$$
\Pi_{t}\left(\widetilde{\chi}_{q}\right)=\chi_{q}^{L}\left(V^{L}\right) \quad \text { and } \quad \Pi_{q}\left(\tilde{\chi}_{q}\right)=\chi_{q}(V) .
$$

Besides, we say that $\tilde{\chi}_{q}$ is an interpolating $(q, t)$-character of $V$.

A given representation $V$ may have different Langlands dual representations (for example, obtained by a shift of the spectral parameter by $t^{N}$, that is by replacing each $Z_{i, a_{i}^{r}}$ by $Z_{i,\left(a t^{N}\right)_{i}^{r_{i}^{\vee}}}$ in $\left.\chi_{t}^{L}\left(V^{L}\right)\right)$. Following [4,15], we will call a representation which cannot be factorized as a tensor product of non-trivial representations, a prime representation.

CONJECTURE 3.10. Any irreducible representation $V$ of $U_{q}(\widehat{\mathfrak{g}})$ has a Langlands dual representation $V^{L}$. Moreover, if $V$ is prime, $V^{L}$ is unique up to a shift of spectral parameter.

If $V$ is not prime, the uniqueness statement does not necessarily hold (see Remark 4.19). Conjecture 3.10 implies Conjecture 2.2 as the condition $\widetilde{M} \in \widetilde{\mathcal{Y}}_{q, t} \backslash$ $\alpha \widetilde{\mathcal{Y}}_{q, t}$ implies that the highest weight of $V^{L}$ is given by the highest weight of $V$. The following is the main result of this paper:

THEOREM 3.11. Let $V$ be a KR module over $U_{q}(\widehat{\mathfrak{g}})$ or an irreducible tensor product of KR modules. Then $V$ has a Langlands dual representation. Moreover, the Langlands dual representation of a KR module over $U_{q}(\widehat{\mathfrak{g}})$ is a KR module over $U_{t}\left({ }^{L} \widehat{\mathfrak{g}}\right)$.

To prove this Theorem, we will use the affine-minuscule property of the KR modules.

\subsection{EXAMPLES}

Let us give some examples of interpolating $(q, t)$-characters which will be useful in the following proofs: 
First consider the type $A_{1}$ with $r=1$. We choose $\phi(1)=0$ and we have the following:

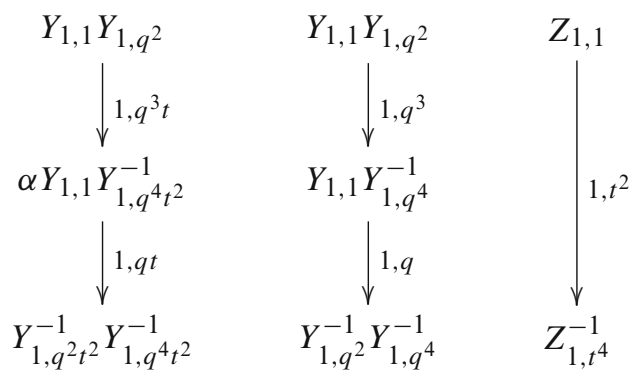

Here we use diagrammatic formulas for (interpolating) $q$-characters as defined in [8]. The left term is the interpolating $q$-character, and then we have the respective specializations at $t=1$ and $q=\epsilon$.

Consider the type $A_{1}$ with $r=2$. We choose $\phi(1)=0$ and we have the following.

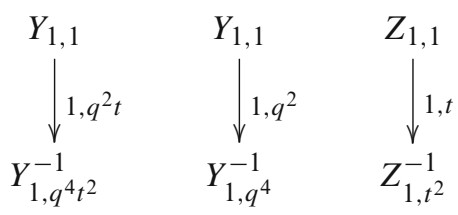

Next, consider the type $A_{2}$ with $r=1$. We choose $\phi(1)=0, \phi(2)=1$ and we have the following:
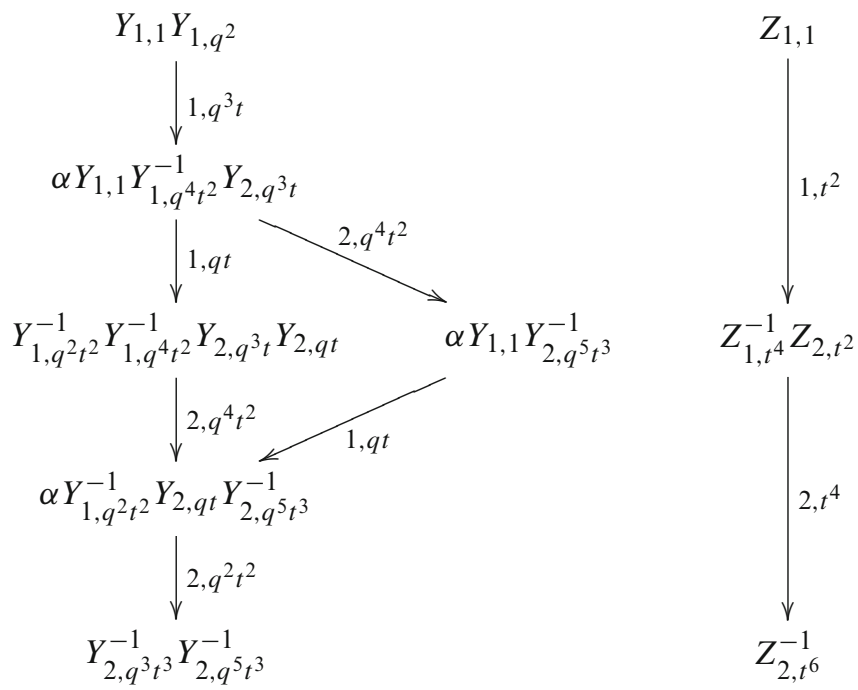
For the type $A_{2}$ with $r=2$, we choose $\phi(1)=0, \phi(2)=1$ and we have the following:

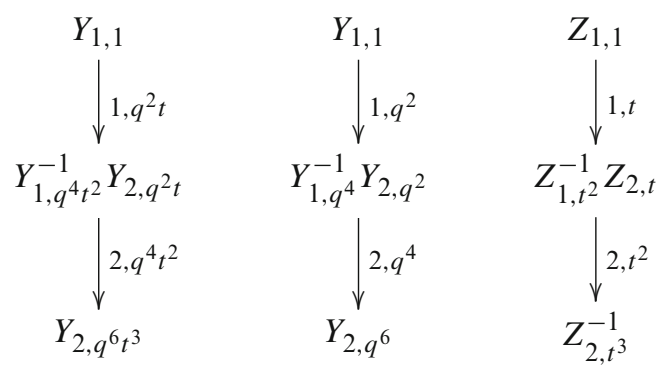

The following example was considered in [7] (it is rewritten here in the language of $q$-characters and twisted $t$-characters): The type is $B_{2}^{(1)}=C_{2}^{(1)}$ and its Langlands dual $D_{3}^{(2)}=A_{3}^{(2)}$. We have $\phi(1)=0, \phi(2)=1$. $\Pi_{q}$ gives the $q$-character of a fundamental 5-dimensional representation of $U_{q}\left(C_{2}^{(1)}\right)$ from [6] (see also [18]) and $\Pi_{t}$ gives the following interpolating $(q, t)$-character:
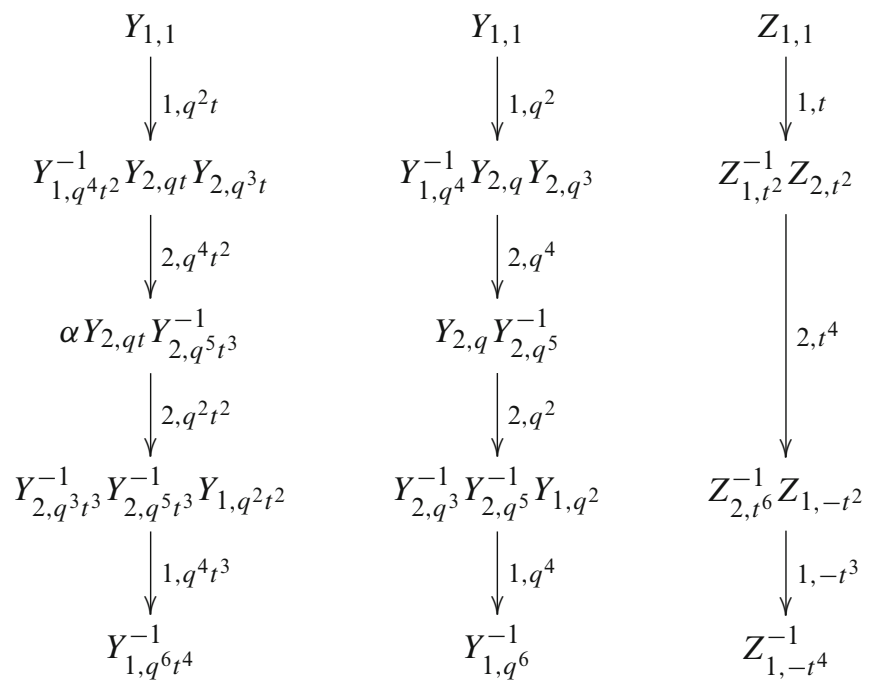

By [13] this is the twisted $t$-character of a fundamental 4-dimensional representation of $U_{t}\left(A_{3}^{(2)}\right)$. 
Let us give another example for this type.

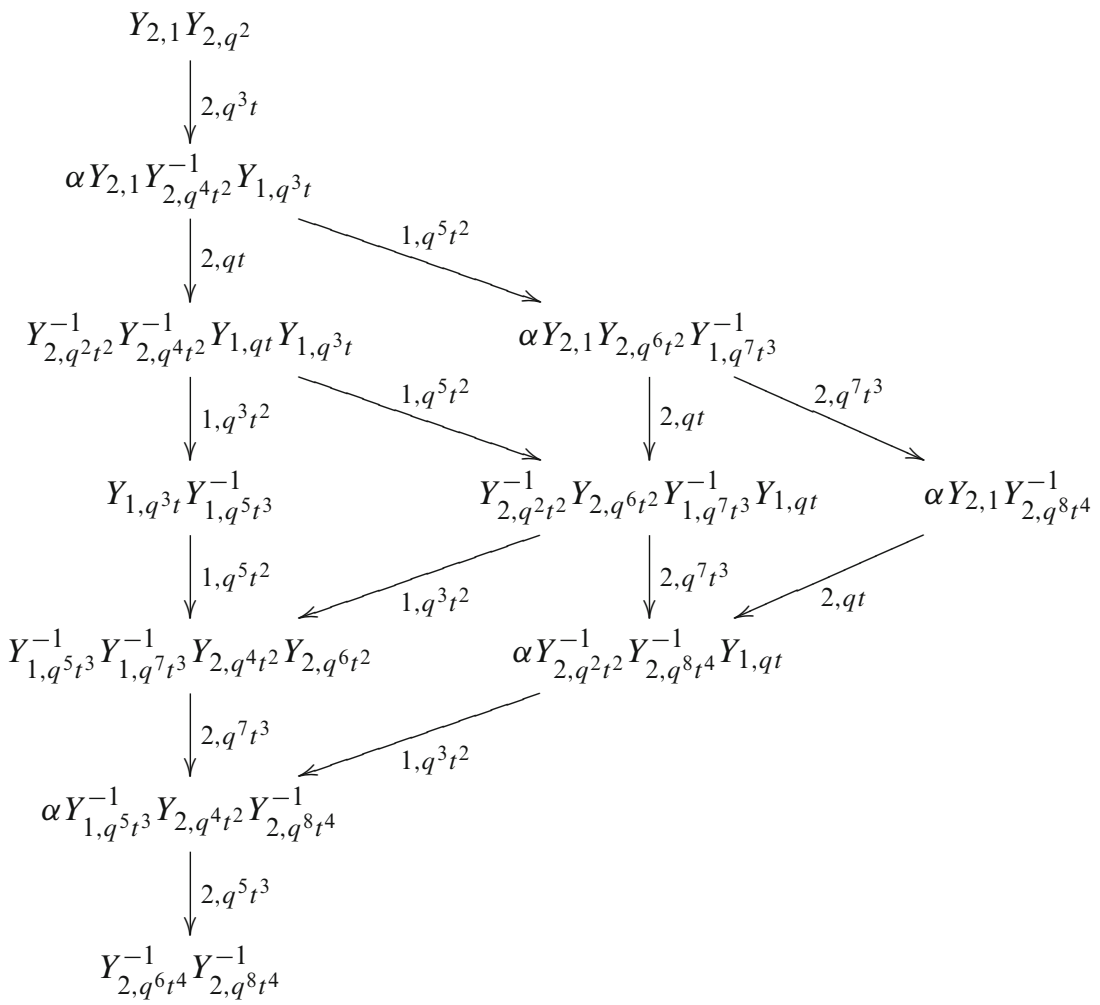

Here we have to check that it is in the $\mathfrak{K}$, since a priori it is unclear that

$$
\alpha Y_{2,1} Y_{2, q^{6} t^{2}} Y_{1, q^{7} t^{3}}^{-1}+Y_{2, q^{2} t^{2}}^{-1} Y_{2, q^{6} t^{2}} Y_{1, q^{7} t^{3}}^{-1} Y_{1, q t}+\alpha Y_{2,1} Y_{2, q^{8} t^{4}}^{-1}+\alpha Y_{2, q^{2} t^{2}}^{-1} Y_{2, q^{8} t^{4}}^{-1} Y_{1, q t}
$$

is in $\mathfrak{K}_{2, q, t}$. But if we subtract $\alpha Y_{2,1}\left(1+A_{2, q t}^{-1}\right) Y_{2, q^{6} t^{2}}\left(1+A_{2, q^{7} t^{3}}^{-1}\right) Y_{1, q^{7} t^{3}}^{-1} \in \mathfrak{K}_{2, q, t}$, we get

$$
(1-\alpha) Y_{2, q^{2} t^{2}}^{-1} Y_{2, q^{6} t^{2}} Y_{1, q^{7} t^{3}}^{-1} Y_{1, q t}=(1-\alpha) Y_{1, q^{7} t^{3}}^{-1} Y_{1, q t} \in \mathfrak{K}_{2, q, t}
$$

$\Pi_{q}$ gives the $q$-character of a 11 -dimensional $\mathrm{KR}$ module over $U_{q}\left(B_{2}^{(1)}\right)$ (it follows from [11] that the formula of $[16,17]$ is satisfied) and $\Pi_{t}$ gives the following: 


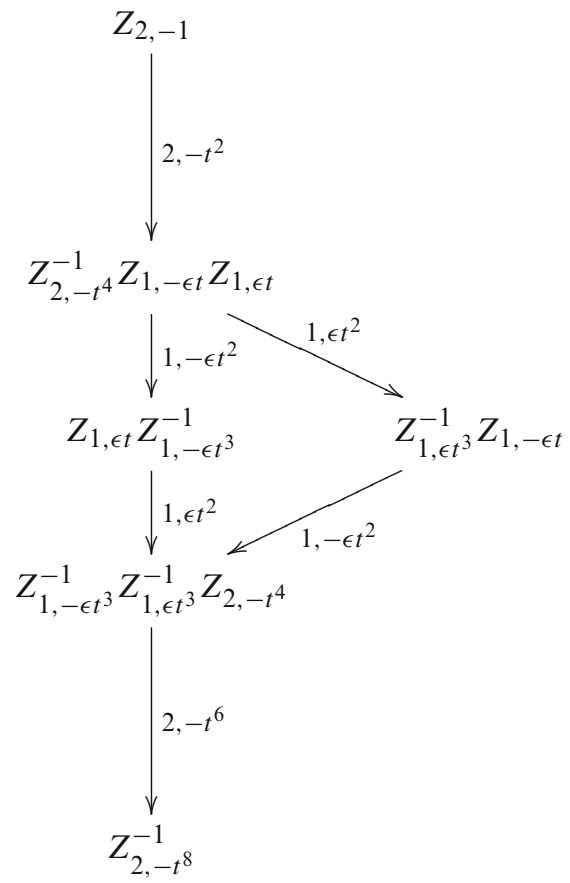

By [13] this is the twisted $t$-character of a fundamental 6-dimensional representation of $U_{t}\left(A_{3}^{(2)}\right)$.

\section{Interpolating $(q, t)$-Characters}

In this section we construct interpolating $(q, t)$-characters in a systematic way: we prove the existence and construct sums in $\mathfrak{K}$ with a unique dominant monomial which can be seen as interpolating $(q, t)$-characters of virtual representations (Theorem 4.4). Their existence implies Conjecture 3.10 in many cases. We will prove in Section 4.3 that Theorem 4.4 implies Theorem 3.11.

Let us explain the main ideas of the construction of interpolating $(q, t)$-characters. In [9, Section 5] a process is given to construct some deformations of $q$-characters. Although the notion of "interpolating $(q, t)$-characters" considered in the present paper is completely different from that of the " $q, t$-characters" in [9], we use an analogous process (note that the " $q, t$-characters" of [9] were first introduced in [20] for simply-laced affine quantum algebras by a different method). In fact, the process of [9] may be seen as a general process to produce $t$-deformations under certain conditions. It is based on an algorithm which is analogous to the Frenkel-Mukhin algorithm for $q$-characters [6].

Let us give the main points of the construction. We define a certain property $P(n)$ depending on the rank $n$ of the Lie algebra which means the existence of interpolating $(q, t)$-characters in $\mathfrak{K}$. To prove it by induction on $n$, assuming the existence for the fundamental representations, we first construct some elements 
$E(m)$ which are analogues of interpolating $(q, t)$-characters for standard modules (tensor products of fundamental representations). Then we have three additional steps:

Step 1: we prove $P(1)$ and $P(2)$ using a more precise property $Q(n)$ such that $Q(n)$ implies $P(n)$. The property $Q(n)$ has the following advantage: it can be checked by computation in elementary cases $n=1,2$.

Step 2: we give some consequences of $P(n)$ which will be used in the proof of $P(r)$ $(r>n)$.

Step 3: we prove $P(n)(n \geq 3)$ assuming that $P(r), r \leq n$ are true. We give an algorithm to construct explicitly the interpolating $(q, t)$-characters by using ideas of [9]. As we do not know a priori that the algorithm is well-defined in the general case, we have to show that it never fails. This is a consequence of $P(2)$ as it suffices to check the compatibility conditions for pairs of nodes of the Dynkin diagram. Finally, we prove that the algorithm stops, that is to say it gives a finite sum which makes sense in $\mathfrak{K}$.

\subsection{STATEMENT}

In this section we prove, for $m \in B$, the existence of an element $F(m) \in \mathfrak{K}$ such that $m$ is the unique dominant monomial of $F(m)$. This will imply Theorem 3.11.

We have a partial ordering on the monomials of $\widetilde{\mathcal{Y}}_{q, t}$ :

$$
m \leq m^{\prime} \Leftrightarrow m\left(m^{\prime}\right)^{-1} \in \mathbb{Z}\left[\widetilde{A}_{i, a}^{-1}, \alpha\right]_{i \in I, a \in \mathcal{C}} .
$$

LEMMA 4.1. A non-zero $\chi$ in $\mathfrak{K}_{i, q, t}$ has at least one $i$-dominant monomial.

Proof. Take a monomial $m$ in $\chi$ maximal for the partial ordering $\leq$. It occurs in a product of generators of $\mathfrak{K}_{i, q, t}$, whose product $M$ of highest monomials are greater or equal to $m$ for the partial ordering, that is, $\mathrm{Mm}^{-1}$ is a product of $v(M)$ factors $\widetilde{A}_{i, a}^{-1}$. Let $N$ be the maximal $v(M)$. We suppose that we have written $\chi$ so that $N$ is minimal. If $N=0$, one of the products $M$ is equal to $m$, so $m$ is $i$-dominant. Otherwise, $N>0$. The products $M$ such that $v(M)=N$ should cancel as $m$ is maximal in $\chi$. But the only case where generators of $\mathfrak{K}_{i, q, t}$ have the same highest monomial is when $i \in I_{1}$ as the dominant monomial $\alpha Y_{i, a} Y_{i, a q^{2}}$ is the highest monomial of

$$
\alpha Y_{i, a}\left(1+\widetilde{A}_{i, a q t}^{-1}\right) \alpha Y_{i, a q^{2}}\left(1+\widetilde{A}_{i, a q^{3} t}^{-1}\right)
$$

and of

$$
\alpha Y_{i, a} Y_{i, a q^{2}}\left(1+\alpha \widetilde{A}_{i, a q^{3} t}^{-1}+\widetilde{A}_{i, a q^{3} t}^{-1} \widetilde{A}_{i, a q t}^{-1}\right) .
$$


But the difference of the two is $\alpha Y_{i, a} Y_{i, a q^{2}} \widetilde{A}_{i, a q t}^{-1}=\alpha \prod_{j \sim i} Y_{j, a q}$ in $\tilde{\mathcal{Y}}_{a, t}$. This monomial is $i$-dominant in $\mathfrak{K}_{i, q, t}$ and strictly lower than $\alpha Y_{i, a} Y_{i, a q^{2}}$. So we can rewrite the expression in such a way that the new maximum of the $v(M)$ is strictly lower than $N$. This is a contradiction.

For $J \subset I$, let $\mathfrak{g}_{J}$ be the semi-simple Lie algebra of Cartan Matrix $\left(C_{i, j}\right)_{i, j \in J}$ and $U_{q}(\widehat{\mathfrak{g}})_{J}$ the associated quantum affine algebra with coefficient $\left(r_{i}\right)_{i \in J}$.

As above, by considering a maximal monomial for the partial ordering, we get the following:

LEMMA 4.2. A non-zero element of $\mathfrak{K}_{J}$ has at least one $J$-dominant monomial.

For a monomial $m$ there is a finite number of monomial $m^{\prime} \in m \mathbb{Z}\left[\widetilde{A}_{i, a}^{-1}\right]_{a \in \mathcal{C}}$ which are $i$-dominant. Let $m=\prod_{i \in I, a \in \mathcal{C}} Y_{i, a}^{u_{i, a}(m)}$ and let $C(m)=\left\{a \in \mathcal{C} \mid \exists i \in I, u_{i, a}(m) \neq 0\right\}$. Then we set

$$
D(m)=\left\{m \widetilde{A}_{i_{1}, a_{1}}^{-1} \cdots \widetilde{A}_{i_{N}, a_{N}}^{-1} \mid N \geq 0, i_{j} \in I, a_{j} \in C(m) q^{\mathbb{N}^{*}} t^{\mathbb{N}^{*}}\right\} .
$$

Note that $D(m)$ is countable, any $m^{\prime} \in D(m)$ satisfies $m^{\prime} \leq m$, and $D\left(m^{\prime}\right) \subset D(m)$. Finally, set

$$
\widetilde{D}(m)=\oplus_{m^{\prime} \in D(m)} \mathbb{Z} m^{\prime} .
$$

We prove the following result as in [9, Lemma 3.14]:

LEMMA 4.3. For any monomial $m$, the set $D(m) \cap B$ is finite.

Let us state the main result of this section.

THEOREM 4.4. For all $n \geq 1$ we have the following property $P(n)$ : for all semi-simple Lie algebras $\mathfrak{g}$ of rank $r k(\mathfrak{g})=n$ and for all $m \in B$ there is a unique $F(m) \in \mathfrak{K} \cap$ $\tilde{D}(m)$ such that $m$ is the unique dominant monomial of $F(m)$.

Remark 4.5. If $m$ is of the form $\alpha m^{\prime}$, then the existence of $F(m)$ follows from the analogous result for the $q$-characters. Indeed, in [9] an algorithm inspired by the Frenkel-Mukhin algorithm [6] was proposed (as well as its $t$-deformation in the sense of [9]): if it is well-defined, then for a dominant monomial $m \in \mathbb{Z}\left[Y_{i, q^{r}}\right]_{i \in I, r \in \mathbb{Z}}$ it gives $F(m)$ in the ring of $q$-character such that $m$ is the unique dominant monomial of $F(m)$ (see also [10]). As a consequence, it suffices to prove the result when $m$ is a product of the $W_{i, a}$. 


\subsection{PROOF OF THEOREM 4.4}

First note that for $n=1$ we have already proved this result. For a general $n$, the uniqueness follows from lemma 4.2.

First, we define a new property $Q(n)$.

DEFINITION 4.6. For $n \geq 1$ denote by $Q(n)$ the property "for all semi-simple Lie algebras $\mathfrak{g}$ of rank $n$, for all $i \in I$ there is a unique $F\left(W_{i, 1}\right) \in \mathfrak{K} \cap \widetilde{D}\left(W_{i, 1}\right)$ such that $W_{i, 1}$ is the unique dominant monomial of $F\left(W_{i, 1}\right)$."

\subsubsection{Construction of the $E(m)$}

We suppose that for $i \in I$, there is $F\left(W_{i, 1}\right) \in \mathfrak{K} \cap \widetilde{D}\left(W_{i, 1}\right)$ such that $W_{i, 1}$ is the unique dominant monomial of $F\left(W_{i, 1}\right)$ (that is the property $Q(n)$ is satisfied).

For $a \in \mathcal{C}$ consider $s_{a}: \widetilde{\mathcal{Y}}_{q, t} \rightarrow \widetilde{\mathcal{Y}}_{q, t}$ the algebra morphism such that $s_{a}\left(Y_{j, b}\right)=Y_{j, a b}$. We can define for $m=\prod_{i \in I, a \in \mathcal{C}} W_{i, a}^{w_{i, a}}$ the element

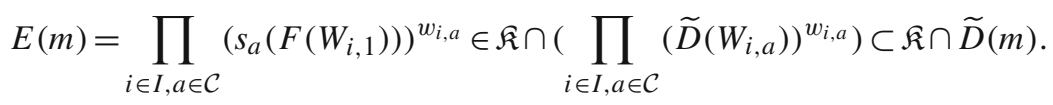

\subsubsection{Step 1}

First, we prove that $Q(n)$ implies $P(n)$.

LEMMA 4.7. For $n \geq 1$, property $Q(n)$ implies property $P(n)$.

Proof. We suppose that $Q(n)$ is true. In particular, we can construct $E(m) \in \mathfrak{K} \cap$ $\widetilde{D}(m)$ for $m \in B$ as above. Let us prove $P(n)$. Let $m \in B$. The uniqueness of $F(m)$ follows from Lemma 4.2. Let $m_{L}=m>m_{L-1}>\cdots>m_{1}$ be the dominant monomials of $D(m)$ with a total ordering compatible with the partial ordering (it follows from Lemma 4.3 that $D(m) \cap B$ is finite). Let us prove by induction on $l$ the existence of $F\left(m_{l}\right)$. The unique dominant monomial of $D\left(m_{1}\right)$ is $m_{1}$, so $F\left(m_{1}\right)=$ $E\left(m_{1}\right) \in \widetilde{D}\left(m_{1}\right)$. In general, let $\lambda_{1}, \ldots, \lambda_{l-1} \in \mathbb{Z}$ be the coefficient of the dominant monomials $m_{1}, \ldots, m_{l-1}$ in $E\left(m_{l}\right)$. We put

$$
F\left(m_{l}\right)=E\left(m_{l}\right)-\sum_{r=1 \cdots l-1} \lambda_{r} F\left(m_{r}\right) .
$$

It follows from the construction that $F(m) \in \widetilde{D}(m)$ because for $m^{\prime} \in D(m)$ we have $E\left(m^{\prime}\right) \in \widetilde{D}\left(m^{\prime}\right) \subseteq \widetilde{D}(m)$.

COROLLARY 4.8. The properties $Q(1), Q(2)$, and hence $P(1), P(2)$, are true.

This allows us to start our induction in the proof of Theorem 4.4. 
Proof. For $n=1$ we have two cases $A_{1}$ with $r=1$ or $r=2$. The explicit formulas have been given above. For $n=2$ we have five cases $A_{1} \times A_{1}$ with $r=1,2, A_{2}$ with $r=1,2), B_{2}$. The cases $A_{1} \times A_{1}$ are a direct consequence of the case $n=1$. For $A_{2}$, $i=1,2$ are symmetric so it suffices to give the formulas for $i=1$ as we did above. We also gave the formulas for $B_{2}$ above.

\subsubsection{Step 2}

Let be $n \geq 1$. We suppose in this section that $P(n)$ is proved. We give some consequences of $P(n)$ which will be used in the proof of $P(r)(r>n)$.

From Lemma 4.3 , an element of $\widetilde{\mathcal{Y}}_{q, t}$ has a finite number of dominant monomials.

PROPOSITION 4.9. We suppose $r k(\mathfrak{g})=n$. We have

$$
\mathfrak{K}=\bigoplus_{m \in B} \mathbb{Z} F(m)
$$

Proof. Let $\chi \in \mathfrak{K}$. Let $m_{1}, \ldots, m_{L} \in B$ the dominant monomials occurring in $\chi$ and $\lambda_{1}, \ldots, \lambda_{L} \in \mathbb{Z}$ their coefficients. It follows from Lemma 4.2 that $\chi=$ $\sum_{l=1 \cdots L} \lambda_{l} F\left(m_{l}\right)$.

COROLLARY 4.10. We suppose $|I|>n$ and let $J \subset I$ such that $|J|=n$. For $m \in$ $B_{J}$, there is a unique $F_{J}(m) \in \mathfrak{K}_{J}$ such that $m$ is the unique $J$-dominant monomial of $F_{J}(m)$. Moreover, $F_{J}(m) \in \widetilde{D}(m)$ and we have

$$
\mathfrak{K}_{J}=\bigoplus_{m \in B_{J}} \mathbb{Z} F_{J}(m)
$$

Proof. The uniqueness of $F(m)$ follows from Lemma 4.2. Let us write $m=m_{J} m^{\prime}$ where $m_{J}=\prod_{i \in J, l \in \mathbb{Z}} Y_{i, l}^{u_{i, l}(m)} \in B_{J}$. In particular, Proposition 4.9 with the algebra $U_{q}(\widehat{\mathfrak{g}})_{J}$ of rank $n$ gives $m_{J} \chi$, where $\chi$ is a polynomial in the variable $\widetilde{A}_{i, l}^{-1}$ for $U_{q}(\widehat{\mathfrak{g}})_{J}$. It suffices to put $F_{J}(m)=m v_{J}(\chi)$, where $v_{J}$ is the ring morphism which sends a variable $\widetilde{A}_{i, a}^{-1}$ for $U_{q}(\widehat{\mathfrak{g}})_{J}$ to the corresponding variable for $U_{q}(\widehat{\mathfrak{g}})$. The last assertion is proved as in Proposition 4.9.

\subsubsection{Step 3}

We explain why properties $P(r)(r<n)$ imply $P(n)$. In particular, we define an algorithm which constructs explicitly the $F(m)$ by using ideas of [9].

We prove the property $P(n)$ by induction on $n \geq 1$. We have proved $P(1)$ and $P(2)$. Let $n \geq 3$ and suppose that $P(r)$ is proved for $r<n$. 
Let $m_{0} \in B$ and $m_{0}, m_{1}, m_{2}, \ldots$ the countable set $D\left(m_{0}\right)$ with indexes such that $m_{j} \geq m_{j^{\prime}}$ implies $j^{\prime} \geq j$.

For $J \varsubsetneqq I$ and $m \in B_{J}$, it follows from $P(r)$ and Corollary 4.10 that there is a unique $F_{J}(m) \in \widetilde{D}(m) \cap \mathfrak{K}_{J}$ such that $m$ is the unique $J$-dominant monomial of $F_{J}(m)$ and that $\mathfrak{K}_{J}=\bigoplus_{m \in B_{J}} \mathbb{Z} F_{J}(m)$. If $m \notin B_{J}$, we denote $F_{J}(m)=0$. For $\chi \in \widetilde{\mathcal{Y}}_{q, t}$, $[\chi]_{m^{\prime}} \in \mathbb{Z}$ is the coefficient of $m^{\prime}$ in $\chi$.

We consider the following inductive definition of the sequences: $\left(s\left(m_{r}\right)\right)_{r \geq 0} \in \mathbb{Z}^{\mathbb{N}}$, $\left(s_{J}\left(m_{r}\right)\right)_{r \geq 0} \in \mathbb{Z}^{\mathbb{N}}(J \varsubsetneqq I)$,

$$
s\left(m_{0}\right)=1, \quad s_{J}\left(m_{0}\right)=0,
$$

and for $r \geq 1, J \subsetneq I$,

$$
\begin{aligned}
s_{J}\left(m_{r}\right) & =\sum_{r^{\prime}<r}\left(s\left(m_{r^{\prime}}\right)-s_{J}\left(m_{r^{\prime}}\right)\right)\left[F_{J}\left(m_{r^{\prime}}\right)\right]_{m_{r}}, \\
s\left(m_{r}\right) & = \begin{cases}s_{J}\left(m_{r}\right) & \text { if } m_{r} \notin B, \\
0 & \text { if } m_{r} \in B .\end{cases}
\end{aligned}
$$

The definition of $s_{J}$ means that we add the various contributions of the $m_{r^{\prime}}$ where $r^{\prime}<r$ with coefficient $\left(s\left(m_{r^{\prime}}\right)-s_{J}\left(m_{r^{\prime}}\right)\right)$, so that a contribution is not counted twice. For the definition of $s\left(m_{r}\right)$, there is something to be proved: that the various $s_{J}\left(m_{r}\right)$ for $m_{r} \notin B_{J}$ coincide.

We prove that the algorithm defines sequences in a unique way. We see that if $s\left(m_{r}\right), s_{J}\left(m_{r}\right)$ are defined for $r \leq R$, then so are $s_{J}\left(m_{R+1}\right)$ for $J \subsetneq I$. Moreover, $s_{J}\left(m_{R}\right)$ imposes the value of $s\left(m_{R+1}\right)$, and by induction the uniqueness is clear. We say that the algorithm is well-defined to step $R$ if there exist $s\left(m_{r}\right), s_{J}\left(m_{r}\right)$ such that the formulas of the algorithm are satisfied for $r \leq R$.

LEMMA 4.11. The algorithm is well-defined to step $r$ if and only if

$$
\forall J_{1}, J_{2} \varsubsetneqq I, \forall r^{\prime} \leq r,\left(m_{r^{\prime}} \notin B_{J_{1}} \text { and } m_{r^{\prime}} \notin B_{J_{2}} \Rightarrow s_{J_{1}}\left(m_{r^{\prime}}\right)=s_{J_{2}}\left(m_{r^{\prime}}\right)\right) \text {. }
$$

Proof. If for $r^{\prime}<r$ the $s\left(m_{r^{\prime}}\right), s_{J}\left(m_{r^{\prime}}\right)$ are well-defined, so is $s_{J}\left(m_{r}\right)$. If $m_{r} \in B$, $s\left(m_{r}\right)=0$ is well-defined. If $m_{r} \notin B$, it is well-defined if and only if $\left\{s_{J}\left(m_{r}\right) \mid m_{r} \notin B_{J}\right\}$ has a unique element.

If the algorithm is well-defined to step $r$, then for $J \varsubsetneqq I$ we set

$$
\mu_{J}\left(m_{r}\right)=s\left(m_{r}\right)-s_{J}\left(m_{r}\right), \quad \chi_{J}^{r}=\sum_{r^{\prime} \leq r} \mu_{J}\left(m_{r^{\prime}}\right) F_{J}\left(m_{r^{\prime}}\right) \in \mathfrak{K}_{J} .
$$

We prove as in [9, Lemma 5.21] (except that the coefficients are in $\mathbb{Z}$ and not in $\left.\mathbb{Z}\left[t^{ \pm 1}\right]\right)$ the following: 
LEMMA 4.12. If the algorithm is well-defined to step $r$, for $J \subset I$ we have

$$
\chi_{J}^{r} \in\left(\sum_{r^{\prime} \leq r} s\left(m_{r^{\prime}}\right) m_{r^{\prime}}\right)+s_{J}\left(m_{r+1}\right) m_{r+1}+\sum_{r^{\prime}>r+1} \mathbb{Z} m_{r^{\prime}}
$$

For $J_{1} \subset J_{2} \subsetneq I$, we have

$$
\chi_{J_{2}}^{r}=\chi_{J_{1}}^{r}+\sum_{r^{\prime}>r} \lambda_{r^{\prime}} F_{J_{1}}\left(m_{r^{\prime}}\right)
$$

where $\lambda_{r^{\prime}} \in \mathbb{Z}$. In particular, if $m_{r+1} \notin B_{J_{1}}$, we have $s_{J_{1}}\left(m_{r+1}\right)=s_{J_{2}}\left(m_{r+1}\right)$.

We prove as in [9, Lemma 5.22] the following:

LEMMA 4.13. The algorithm never fails.

Now we aim at proving that the algorithm stops. We will use the following notion [6]:

DEFINITION 4.14. A non-trivial $m=\prod_{i \in I, a \in \mathbb{C}^{\times}} Y_{i, a}^{u_{i, a}(m)}$ is said to be rightnegative if for all $a \in \mathbb{C}^{\times}, j \in I$ we have $\left(u_{j, a q^{L_{a}}}(m) \neq 0 \Rightarrow u_{j, a q^{L_{a}}}(m)<0\right)$ where

$$
L_{a}=\max \left\{l \in \mathbb{Z} \mid \exists i \in I, u_{i, a q} L(m) \neq 0\right\} .
$$

$D(m)$ is graded by finite-dimensional subspaces such that the degree of the monomial $m^{\prime}=m \widetilde{A}_{i_{1}, a_{1}}^{-1} \cdots \widetilde{A}_{i_{N}, a_{N}}^{-1}$ in $D(m)$ is $N$. Then we can consider the corresponding graded completion $\bar{D}(m)$ of $\widetilde{D}(m)$. By an infinite sum in $\tilde{\mathcal{Y}}_{q, t}$ we mean an element in such a completion. We have analogous definitions for infinite sums in $\mathcal{Y}_{q}$ and in $\mathcal{Y}_{t}^{L}$

LEMMA 4.15. Let $S$ be an infinite sum in $\mathcal{Y}_{q}$ (resp. in $\mathcal{Y}_{t}^{L}$ ) which is an infinite sum of elements in $\mathfrak{K}_{i, q}$ (resp. in $\mathfrak{K}_{i, t}^{L}$ ) for any $i \in I$. If $S$ contains a finite number of dominant monomials, then $S$ is a finite sum in $\mathcal{Y}_{q}$ (resp. in $\mathcal{Y}_{t}^{L}$ ).

Proof. We prove the result for $\mathcal{Y}_{q}$ (the proof is completely analogous for $\mathcal{Y}_{t}^{L}$ by using results in [13]). Let $m_{1}, \ldots, m_{L}$ be the dominant monomials occurring in $S$ and $\lambda_{1}, \ldots, \lambda_{L}$ their multiplicity. For $m$ a dominant monomial, there is $F_{q}(m) \in$ $\operatorname{Im}\left(\chi_{q}\right)$ with a unique dominant monomial $m$ (see the construction in $[9$, Section 5.1] by using $q$-characters which are finite sums). Then

$$
S^{\prime}=S-\sum_{1 \leq l \leq L} \lambda_{l} F_{q}\left(m_{l}\right)
$$


has no dominant monomial and for any $i \in I$ is an infinite sum of elements in $\mathfrak{K}_{i, q}$. So if $S^{\prime} \neq 0$, a maximal monomial occurring in $S^{\prime}$ is dominant, contradiction. So $S^{\prime}=0$.

Now we can prove the following:

LEMMA 4.16. The algorithm stops and $\chi=\sum_{r \geq 0} s\left(m_{r}\right) m_{r} \in \mathfrak{K} \cap \widetilde{D}\left(m_{0}\right)$. Moreover, the only dominant monomial in $\chi$ is $m_{0}$.

Proof. Consider the (a priori, non necessarily finite) sum $\chi$ in $\bar{D}\left(m_{0}\right)$. We prove as in [9, Lemma 5.23] that for each $i \in I, \chi$ is an infinite sum of elements in $\mathfrak{K}_{i, q, t}$.

There in $N \in \mathbb{Z}$ such that $m_{0} \in \mathbb{Z}\left[Y_{i, q^{r} t^{l}}\right]_{i \in I, r, l \leq N}$. By construction with the algorithm, only a finite number of monomials of $F\left(m_{0}\right)$ are in $m_{0} \mathbb{Z}\left[\tilde{A}_{i, q^{r} t^{l}}^{-1}\right]_{r \leq N}$ or $l \leq N$. Let us consider another monomials $m^{\prime} \notin \mathbb{Z}\left[\tilde{A}_{i, q^{r} t^{\prime}}^{-1}\right]_{r \leq N}$ or $l \leq N$ occurring in $\chi$. The specializations $\Pi_{q}\left(m^{\prime}\right)$ and $\Pi_{t}\left(m^{\prime}\right)$ are right-negative. Indeed for any $r_{1}, r_{2}>N$ and

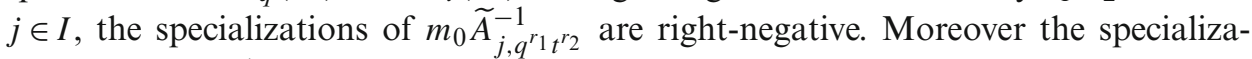
tions of the $\widetilde{A}_{i, a}^{-1}$ are right-negative, and a product of right-negative monomials is right-negative [6]. Since a right-negative monomial is not dominant, we can conclude that the specializations of $m^{\prime}$ are not dominant. So $\Pi_{q}(\chi)$ and $\Pi_{t}(\chi)$ have a finite number of dominant monomials. So these are finite sums by Lemma 4.15. As $\tilde{\mathcal{Y}}_{q, t}$ is obtained by a quotient by $\operatorname{Ker}\left(\Pi_{q}\right) \cap \operatorname{Ker}\left(\Pi_{t}\right), \chi$ is a finite sum.

This lemma implies the following:

COROLLARY 4.17. For $n \geq 3$, if the $P(r)(r<n)$ are true, then $P(n)$ is true.

In particular, Theorem 4.4 is proved by induction on $n$.

\subsection{PROOF OF THEOREM 3.11}

Let us explain how Theorem 4.4 implies Theorem 3.11.

First consider the dominant interpolating monomial

$$
m=W_{i, a} W_{i, a t^{2} q^{4}} \cdots W_{i, a\left(t^{2} q^{4}\right)^{k-1}} .
$$

The specializations by $\Pi_{q}, \Pi_{t}$ of $m$ correspond to the highest monomials of KR modules, respectively, over $U_{q}(\widehat{\mathfrak{g}})$ and $U_{t}\left({ }^{L} \widehat{\mathfrak{g}}\right)$. By construction, the monomials $m^{\prime}$ occurring in $F(m)-m$ are of the form

$$
m^{\prime}=\left(m \widetilde{A}_{i, a q_{i}^{2 k}}^{-1}\right) \widetilde{A}_{i_{1}, a_{1}}^{-1} \cdots \widetilde{A}_{i_{N}, a_{N}}^{-1} \quad \text { where } i_{1}, \ldots, i_{N} \in I \text { and } a_{1}, \ldots, a_{N} \in \mathcal{C}
$$


As a consequence, $\Pi_{q}\left(m^{\prime}\right)$ and $\Pi_{t}\left(m^{\prime}\right)$ are right-negative. Indeed, the specialization of $m \widetilde{A}_{i, a q_{i}^{2 k-1} t}^{-1}$ and of the $\widetilde{A}_{i, a}^{-1}$ are right-negative, and a product of rightnegative monomials is right-negative [6]. Since a right-negative monomial is not dominant, the specializations of $F(m)$ are affine-minuscule. By Theorem 3.3 and Theorem 3.5, this completes the proof of the first statement of Theorem 3.11 for KR modules.

Now we have the following compatibility property with tensor products:

PROPOSITION 4.18. Let $V_{1}$ and $V_{2}$ be two simple representations of $U_{q}(\hat{\mathfrak{g}})$ with respective Langlands dual representations $V_{1}^{L}$ and $V_{2}^{L}$. If $V_{1} \otimes V_{2}$ is simple, then $V_{1}^{L} \otimes V_{2}^{M}$ is a Langlands dual representation to $V_{1} \otimes V_{2}$.

Proof. For $\chi_{1}$ and $\chi_{2}$ interpolating $(q, t)$-characters, respectively, of $V_{1}$ and $V_{2}$, the product $\chi_{1} \chi_{2}$ is clearly an interpolating $(q, t)$-character of $V_{1} \otimes V_{2}$ as $\mathfrak{K}$ is a subring of $\tilde{\mathcal{Y}}_{q, t}$ and $\Pi_{q}$ is a ring morphism. We can conclude for the last point as $\Pi_{t}$ is a ring morphism.

According to the above discussion, this completes the proof of the first statement of Theorem 3.11.

Remark 4.19. If $V_{1}^{L}$ and $V_{2}^{L}$ are nontrivial, then the uniqueness statement does not hold for $V_{1} \otimes V_{2}$ : by shifting the spectral parameter in $\chi_{1}$ by $t^{N}$ where $N \neq 1$ without changing $\chi_{2}$, we get another Langlands dual representation which cannot be obtained from $V_{1}^{L} \otimes V_{2}^{L}$ by a shift of spectral parameter.

Now consider a KR module $V$ of highest monomial $W_{i, a} W_{i, a q^{4}} \cdots W_{i, a\left(q^{4}\right)^{k-1}}$. Suppose that we have a Langlands dual representation $V^{L}$ which is not a KR module and consider a corresponding interpolating $(q, t)$-character $\chi$. Let $M$ be the highest monomial of $\chi$. Then, there are $b \neq b^{\prime}$ such that $W_{i, b}, W_{i, b^{\prime}}$ occur in $M$ but $W_{i, b q^{4} t^{2}}, W_{i, b^{\prime} q^{4} t^{2}}$ do not occur in $M$. Suppose that $r_{i}=1$ (resp. $r_{i}=2$ ). As a consequence, $M A_{i, b q^{3} t}^{-1} A_{i, b q t}^{-1}$ and $M A_{i, b^{\prime} q^{3} t}^{-1} A_{i, b^{\prime} q t}^{-1}$ (resp. $M A_{i, b q^{2} t}^{-1}$ and $M A_{i, b^{\prime} q^{2} t}^{-1}$ ) occur in $\chi$ and have distinct image by $\Pi_{q}$. But by [11, Lemma 5.5], $\chi_{q}(V)$ contains a unique monomial of the form $\Pi_{q}(M) A_{i, a^{\prime}}^{-1} A_{i, a^{\prime \prime}}^{-1}\left(\operatorname{resp} . \Pi_{q}(M) A_{i, a^{\prime}}^{-1}\right)$. This contradicts $\Pi_{q}(\chi)=\chi_{q}(V)$.

\subsection{ADDITIONAL COMMENTS}

Note that it is easy to construct interpolating $(q, t)$-characters of non-simple representations by using tensor products of KR modules which are not simple, by the same method as in Proposition 4.18. More interestingly, to illustrate Conjecture 3.10, let us give an example of a simple non affine-minuscule module which satisfies the Langlands duality. Consider the $U_{q}\left(C_{2}^{(1)}\right)$-module $V=L\left(Y_{1,1}^{2} Y_{2, q^{5}} Y_{2, q^{7}}\right)$. 
Note that $L\left(Y_{2,1} Y_{2, q^{2}} Y_{1, q^{7}}\right) \otimes L\left(Y_{1, q^{7}}\right)$ is simple as it is affine-minuscule. So by [12, Lemma 4.10], $V \simeq L\left(Y_{1,1} Y_{2, q^{5}} Y_{2, q^{7}}\right) \otimes L\left(Y_{1,1}\right)$. Moreover,

$$
\chi_{q}\left(L\left(Y_{1,1}\right) \otimes L\left(Y_{2, q^{5}} Y_{2, q^{7}}\right)\right)=\chi_{q}\left(L\left(Y_{2,1}\right) \otimes L\left(Y_{2,7}\right)\right)+\chi_{q}\left(L\left(Y_{1,1} Y_{2, q^{5}} Y_{2, q^{7}}\right)\right)
$$

has they have the same multiplicity 1 on the dominant monomials. So

$$
\operatorname{dim}\left(L\left(Y_{2, q^{5}} Y_{2, q^{7}}\right)\right)=55-16=39 \text { and } \operatorname{dim}(V)=39 \times 5=195 .
$$

Now consider the $U_{t}\left(A_{3}^{(2)}\right)$ simple module $V^{L}=L\left(Z_{1,1}^{2} Z_{2, t^{6}}\right)$. In the same way, by [14, Proposition 4.7], we have $V^{L} \simeq L\left(Z_{1,1} Z_{2, t^{6}}\right) \otimes L\left(Z_{1,1}\right)$, and as

$$
\chi_{t}^{\sigma}\left(L\left(Z_{1,1}\right) \otimes L\left(Z_{2, t^{6}}\right)\right)=\chi_{t}^{\sigma}\left(L\left(Z_{1,1} Z_{2, t^{6}}\right)\right)-\chi_{t}^{\sigma}\left(L\left(Z_{1,-t^{2}}\right)\right),
$$

we get $\operatorname{dim}\left(L\left(Z_{1,1} Z_{2, t^{6}}\right)\right)=24-4=20$ and $\operatorname{dim}\left(V^{L}\right)=20 \times 4=80$. As above for the dimension, it is easy to compute the $q$-character (resp. twisted $t$-character) of $V$ $\left(\right.$ resp. $V^{L}$ ). So we can check that $V$ satisfies the Langlands duality with the Langlands dual module $V^{L}$. We do not list the 195 monomial of the interpolating $(q, t)$ character, but the 80 monomials which do not have $\alpha$ in their coefficient. It suffices to multiply one of the 4 monomials of the sum

$$
Y_{1,1}+Y_{1, q^{4} t^{2}}^{-1} Y_{2, q t} Y_{2, q^{3} t}+Y_{2, q^{3} t^{3}}^{-1} Y_{2, q^{5} t^{3}}^{-1} Y_{1, q^{2} t^{2}}+Y_{1, q^{6} t^{4}}^{-1}
$$

by one the following 20 monomials. We use the notation $i_{a}=Y_{i, a}$ (analog notation will also be used in the following):

$$
\begin{aligned}
& 1_{1} 2_{q^{5} t^{3}} 2_{q^{7} t^{3}}, 1_{1} 1_{q^{6} t^{4}} 1_{q^{8} t^{4}} 2_{q^{7} t^{5}}^{-1} 2_{q^{9} t^{5}}^{-1}, 1_{q^{4} t^{2}}^{-1} 2_{q t} 2_{q^{3} t^{3}} 2_{q^{5} t^{3}} 2_{q^{7} t^{3}} 1_{1} 1_{q^{6} t^{4}} 1_{q^{12} t^{6}}^{-1} 2_{q^{7} t^{5}}^{-1} 2_{q^{11} t^{5}} \\
& 1_{1} 1_{q^{10} t^{6}}^{-1} 1_{q^{8} t^{4}}, 1_{q^{4} t^{2}}^{-1} 1_{q^{6} t^{4}} 1_{q^{8} t^{4}} 2_{q t} 2_{q^{3} t^{3}} 2_{q^{7} t^{5}}^{-1} 2_{q^{9} t^{5}}^{-1}, 1_{1} 1_{q^{10} t^{6}}^{-1} 1_{q^{12} t^{6}}^{-1} 2_{q^{9} t^{5}} 2_{q^{11} t^{5}}, 1_{q^{4} t^{2}}^{-1} 1_{q^{10} t^{6}}^{-1} 2_{q t} 2_{q^{3} t^{3}} 1_{q^{8} t^{4}} \text {, } \\
& 1_{q^{4} t^{2}}^{-1} 1_{q^{6} t^{4}} 1_{q^{12} t^{6}}^{-1} 2_{q t} 2_{q^{3} t^{3}} 2_{q^{7} t^{5}}^{-1} 2_{q^{11} t^{5}}, 1_{q^{4} t^{2}}^{-1} 1_{q^{10} t^{6}}^{-1} 1_{q^{12} t^{6}}^{-1} 2_{q t} 2_{q^{3} t} 2_{q^{9} t^{5}} 2_{q^{11} t^{5}}, \\
& 1_{q^{2} t^{2}} 1_{q^{6} t^{4}} 1_{q^{8} t^{4}} 2_{q^{3} t^{3}}^{-1} 2_{q^{5} t^{3}}^{-1} 2_{q^{7} t^{5}}^{-1} 2_{q^{9} t^{5}}^{-1}, 1_{1} 2_{q^{11} t^{7}}^{-1} 2_{q^{13} t^{7}}^{-1}, 1_{q^{2} t^{2}} 1_{q^{10} t^{6}}^{-1} 2_{q^{3} t^{3}}^{-1} 2_{q^{5} t^{3}}^{-1} 1_{q^{8} t^{4}}, \\
& 1_{q^{2} t^{2}} 1_{q^{6} t^{4}} 1_{q^{12} t^{6}}^{-1} 2_{q^{3} t^{3}}^{-1} 2_{q^{5} t^{3}}^{-1} 2_{q^{7} t^{5}}^{-1} 2_{q^{11} t^{5}}, 1_{q^{4} t^{2}}^{-1} 2_{q t^{2}} 2_{q^{3} t} 2_{q^{11} t^{7}}^{-1} q_{q^{13} t^{7}}^{-1}, 1_{q^{2} t^{2}} 1_{q^{10} t^{6}}^{-1} 1_{q^{12} t^{6}}^{-1} 2_{q^{3} t^{3}}^{-1} 2_{q^{5} t^{3}}^{-1} 2_{q^{9} t^{5}} 2_{q^{11} t^{5}} \text {, } \\
& 1_{q^{2} t^{2}} 2_{q^{3} t^{3}}^{-1} 2_{q^{5} t^{3}}^{-1} 2_{q^{11} t^{7}}^{-1} 2_{q^{13} t^{7}}^{-1}, 1_{q^{6} t^{4}}^{-1} 1_{q^{10} t^{6}}^{-1} 1_{q^{8} t^{4}}, 1_{q^{6} t^{4}}^{-1} 1_{q^{10} t^{6}}^{-1} 1_{q^{12} t^{6}}^{-1} 2_{q^{9} t^{5}} 2_{q^{11} t^{6}}, 1_{q^{6} t^{4}}^{-1} 2_{q^{11} t^{7}}^{-1} 2_{q^{13} t^{7}}^{-1} \text {. }
\end{aligned}
$$

Nakajima [20] has computed the $q$-characters of simple modules from those of standard modules (tensor products of fundamental representations) using quiver varieties. His results are not available for non-simply laced untwisted quantum affine algebras, but the second author has conjectured [9] that analogous results do hold in this case. The result on tensor products in Proposition 4.18 is an indication of the compatibility of the two conjectures. 


\subsection{EXAMPLE}

We give an example of an interpolating $(q, t)$-character that we get for a Lie algebra or rank strictly greater than 2 by the process described in the proof. Consider $U_{q}\left(C_{3}^{(1)}\right)$ with $\phi(1)=\phi(3)=0, \phi(2)=1$.

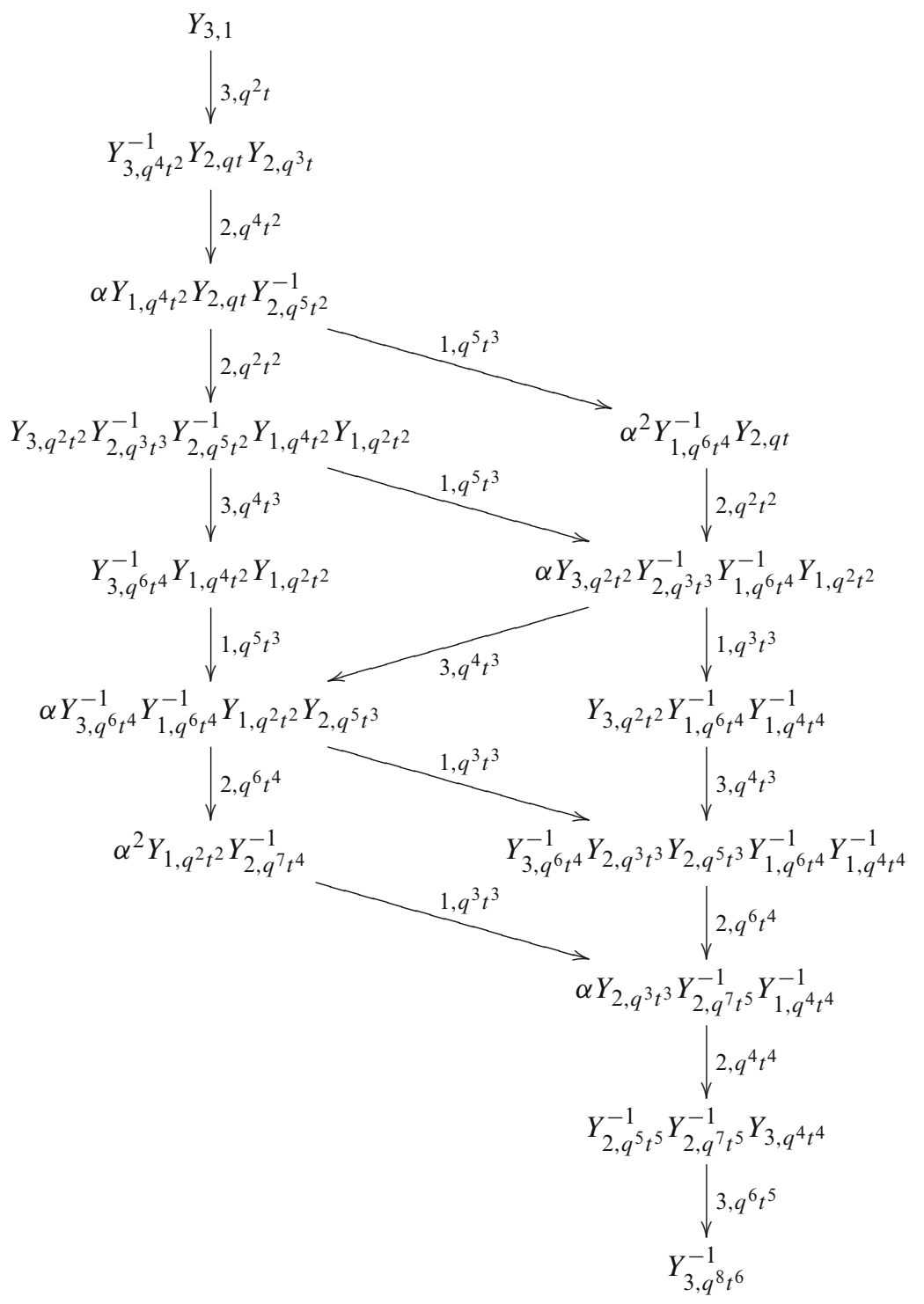

The specialization at $t=1$ gives the $q$-character of a 14-dimensional fundamental representation of $U_{q}\left(C_{3}^{(1)}\right)$ from [6] (see also [18]). The specialization at $q=\epsilon$ gives the twisted $t$-character of a 8-dimensional fundamental representation of $U_{t}\left(D_{5}^{(2)}\right)$ [13]. 


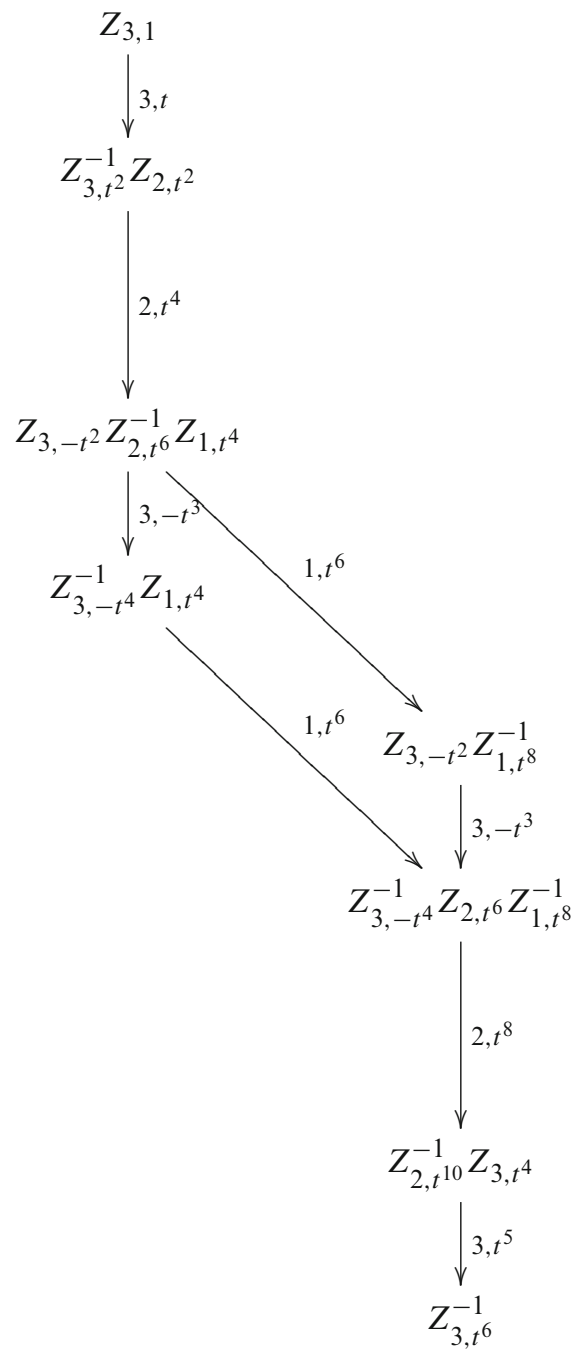

\section{Triple-Laced Case}

Now we suppose that $r=3$, that is to say we consider $U_{q}\left(G_{2}^{(1)}\right)$ and its Langlands dual $U_{t}\left(D_{4}^{(3)}\right)$. The results and their proofs are completely analogous to the case $r=2$, except that we have to change some definitions and formulas and we have to check the existence of interpolating $(q, t)$-characters in some examples as we did for $r=2$.

\subsection{DEFINITIONS OF INTERPOLATING STRUCTURES}

We set $\epsilon=e^{i \pi / 3}$. For the Dynkin diagram of $G_{2}$ we use the convention $r_{1}=3$ and $r_{2}=1$. We have $r_{1}^{\vee}=1$ and $r_{2}^{\vee}=3$. 
For the $q$-characters of $U_{q}\left(G_{2}^{(1)}\right)$ we have

$$
\begin{aligned}
& A_{1, a}=Y_{1, a q^{-3}} Y_{1, a q^{3}} Y_{2, a q^{-2}}^{-1} Y_{2, a}^{-1} Y_{2, a q^{2}}^{-1}, \\
& \mathfrak{K}_{1, q}=\mathbb{Z}\left[Y_{1, a}\left(1+A_{1, a q^{3}}^{-1}\right), Y_{2, a}^{ \pm 1}\right]_{a \in q^{\mathbb{Z}}}, \quad Y_{2, a q^{-1}} Y_{2, a q} Y_{1, a}^{-1}, \\
& \mathfrak{K}_{2, q}=\mathbb{Z}\left[Y_{2, a}\left(1+A_{2, a q}^{-1}\right), Y_{1, a}^{ \pm 1}\right]_{a \in q^{\mathbb{Z}} .}
\end{aligned}
$$

For the twisted $t$-characters of $U_{t}\left(D_{4}^{(3)}\right)$ we have

$$
\begin{aligned}
& B_{1, a}=Z_{1, a t^{-1}} Z_{1, a t} Z_{2, a^{3}}^{-1}, \quad B_{2, a^{3}}=Z_{2, a^{3} t^{3}} Z_{2, a^{3} t^{-3}} Z_{1, a}^{-1} Z_{1, a \epsilon^{2}}^{-1} Z_{1, a \epsilon^{4}}^{-1}, \\
& \mathfrak{K}_{1, t}^{L}=\mathbb{Z}\left[Z_{1, a}\left(1+Z_{1, a t}^{-1}\right), Z_{2, a}^{ \pm 1}\right]_{a \in \epsilon^{\mathbb{Z}} \mathbb{Z}}, \quad \mathfrak{K}_{2, t}^{L}=\mathbb{Z}\left[Z_{2, a}\left(1+A_{2, a t^{3}}^{-1}\right), Z_{1, a}^{ \pm 1}\right]_{a \in \epsilon^{\mathbb{Z}} t^{\mathbb{Z}} .}
\end{aligned}
$$

For $a \in \mathcal{C}$ let $W_{1, a}=Y_{1, a}, W_{2, a}=Y_{2, a q^{-2}} Y_{2, a} Y_{2, a q^{2}}$.

Let us consider an interpolating map $\beta(q, t)$ such that $\beta(q, 1)=1$ and $\beta(\epsilon, t)=0$. We can use, for example, the following map introduced in [1]:

$$
\beta(q, t)=\frac{\left(q^{3}-q^{-3}\right)\left(q t^{-1}-q^{-1} t\right)\left(q^{5} t^{-1}-q^{-5} t\right)\left(q^{4} t^{-2}-q^{-4} t^{2}\right)}{\left(q-q^{-1}\right)\left(q^{3} t^{-1}-q^{-3} t\right)\left(q^{4} t^{-1}-t q^{-4}\right)\left(q^{5} t^{-2}-q^{-5} t^{2}\right)} .
$$

Consider

$$
\mathcal{Y}_{q, t}=\mathbb{Z}\left[W_{i, a}^{ \pm 1}, \beta Y_{i, a}^{ \pm 1}, \beta\right]_{i \in I, a \in \mathcal{C}} .
$$

We have the specializations maps $\Pi_{q}, \Pi_{t}$ and the ideal $\operatorname{Ker}\left(\Pi_{q}\right) \cap \operatorname{Ker}\left(\Pi_{t}\right)$ is generated by the elements

$$
\beta(\beta-1), \beta\left(Y_{i, a}-Y_{i, a t}\right),(\beta-1)\left(W_{i, a q}-W_{i, a \epsilon}\right),\left(W_{i, a}-W_{i, a t}\right)\left(W_{j, b q}-W_{j, b \epsilon}\right),
$$

for $i, j \in I$ and $a, b \in \mathcal{C}$. We work in the ring $\widetilde{\mathcal{Y}}_{q, t}=\mathcal{Y}_{q, t} /\left\langle\operatorname{Ker}\left(\Pi_{q}\right) \cap \operatorname{Ker}\left(\Pi_{t}\right)\right\rangle$.

DEFINITION 5.1. We define for $a \in \mathcal{C}$ the interpolating root monomials

$$
\widetilde{A}_{1, a}=Y_{i, a\left(q^{3} t\right)^{-1}} Y_{i, a q^{3} t}\left(Y_{2, a q^{-2}} Y_{2, a} Y_{2, a q^{2}}\right)^{-1}, \quad \widetilde{A}_{2, a}=Y_{2, a(q t)^{-1}} Y_{2, a q t} Y_{1, a}^{-1} .
$$

We will use the identification $Z_{1, a}=Y_{1, a}$ and $Y_{2, a} Y_{2, \epsilon^{2} a} Y_{2, \epsilon^{4} a}=Z_{2,-a^{3}}$. The $\widetilde{A}_{i, a}$ interpolate between the root monomials of $U_{q}\left(G_{2}^{(1)}\right)$ and $U_{t}\left(D_{4}^{(3)}\right)$ as we have the following:

LEMMA 5.2. We have $\Pi_{q}\left(\widetilde{A}_{i, a}\right)=A_{i, \Pi_{q}(a)}$ for $i \in I, a \in \mathcal{C}$.

We have $\Pi_{t}\left(\widetilde{A}_{2, a q^{-2}} \widetilde{A}_{2, a} \widetilde{A}_{2, a q^{2}}\right)=B_{2,\left(\Pi_{t}(a)\right)^{3}}$ for $a \in \mathcal{C}$.

We have $\Pi_{t}\left(\widetilde{A}_{1, a}\right)=B_{1,-\Pi_{t}(a)}$ for $a \in \mathcal{C}$.

Proof. The first point is clear.

Let $a^{\prime}=\Pi_{q}(a)$. The specialization of $\widetilde{A}_{2, a q^{-2}} \widetilde{A}_{2, a} \widetilde{A}_{i, a q^{2}}$ at $q=\epsilon$ is

$$
\begin{aligned}
& \left(Y_{2,-a^{\prime} t^{-1}} Y_{2,-a^{\prime} \epsilon^{2} t^{-1}} Y_{2,-a^{\prime} \epsilon^{4} t^{-1}}\right)\left(Y_{2,-a^{\prime} t} Y_{2,-a \epsilon^{2} t} Y_{2,-a^{\prime} \epsilon^{4} t}\right) \times\left(Y_{1, a^{\prime} \epsilon^{-2}} Y_{1, a^{\prime}} Y_{1, a^{\prime} \epsilon^{2}}\right)^{-1} \\
& \quad=Z_{2,\left(a^{\prime}\right)^{3} t^{3}} Z_{2,\left(a^{\prime}\right)^{3} t^{-3}} \times\left(Z_{1, a^{\prime} \epsilon^{-2}} Z_{1, a^{\prime}} Z_{1, a^{\prime} \epsilon^{2}}\right)^{-1}=B_{2,\left(a^{\prime}\right)^{3} .}
\end{aligned}
$$


The specialization of $\widetilde{A}_{1, a}$ at $q=\epsilon$ is

$$
Y_{1,-a^{\prime} t^{-1}} Y_{1,-a^{\prime} t} \times\left(Y_{2, a^{\prime} \epsilon^{-2}} Y_{2, a^{\prime}} Y_{2, a^{\prime} \epsilon^{2}}\right)^{-1}=Z_{1,-a^{\prime} t^{-1}} Z_{1,-a^{\prime} t} \times Z_{2,\left(-a^{\prime}\right)^{3}}^{-1}=B_{2,-a^{\prime}}
$$

Consider the following subalgebras of $\widetilde{\mathcal{Y}}_{q, t}$ :

$$
\begin{aligned}
\mathfrak{K}_{1, q, t}= & \mathbb{Z}\left[Y_{1, a}\left(1+\widetilde{A}_{1, a q^{3} t}^{-1}\right), W_{2, a}^{ \pm 1}, \beta Y_{2, a}^{ \pm 1}, \beta\right]_{a \in \mathcal{C}}, \\
\mathfrak{K}_{2, q, t}=\mathbb{Z} & {\left[Y_{2, a} Y_{2, a q^{2}} Y_{2, a q^{4}}\left(1+\beta \widetilde{A}_{2, a q^{5} t}^{-1}+\beta \widetilde{A}_{2, a q^{5} t}^{-1} \widetilde{A}_{2, a q^{3} t}^{-1}+\widetilde{A}_{2, a q^{5} t}^{-1} \widetilde{A}_{2, a q^{3} t}^{-1} \widetilde{A}_{2, a q t}^{-1}\right),\right.} \\
& \left.\beta Y_{2, a}\left(1+\widetilde{A}_{2, a q t}^{-1}\right), Y_{1, a}^{ \pm 1}, \beta\right]_{a \in \mathcal{C}} .
\end{aligned}
$$

These are interpolating subalgebras as

LEMMA 5.3. For $i \in\{1,2\}$, we have $\Pi_{q}\left(\mathfrak{K}_{i, q, t}\right)=\mathfrak{K}_{i, q}$ and $\Pi_{t}\left(\mathfrak{K}_{i, q, t}\right)=\mathfrak{K}_{i, t}^{L}$.

Proof. We have

$$
\begin{aligned}
& \Pi_{q}\left(\mathfrak{K}_{2, q, t}\right)=\mathbb{Z}\left[Y_{2, a}\left(1+A_{2, a q}^{-1}\right), Y_{1, a}^{ \pm 1}\right]_{a \in q^{\mathbb{Z}}}=\mathfrak{K}_{2, q}, \\
& \Pi_{t}\left(\mathfrak{K}_{2, q, t}\right)=\mathbb{Z}\left[Y_{2, a} Y_{2, a \epsilon^{2}} Y_{2, a \epsilon^{4}}\left(1+\widetilde{A}_{2,-a \epsilon^{2} t}^{-1} \widetilde{A}_{2,-a t}^{-1} \widetilde{A}_{2,-a \epsilon^{-2} t}^{-1}\right), Y_{1, a}^{ \pm 1}\right]_{a \in \epsilon^{\mathbb{Z}} t}=\mathfrak{K}_{2, t}^{L},
\end{aligned}
$$

as by Lemma 5.2 we have

$$
Y_{2, a} Y_{2, a \epsilon^{2}} Y_{2, a \epsilon^{4}}\left(1+\widetilde{A}_{2,-a \epsilon^{2} t}^{-1} \widetilde{A}_{2,-a t}^{-1} \widetilde{A}_{2,-a \epsilon^{-2} t}^{-1}\right)=Z_{2,-a^{3}}\left(1+B_{2,-a^{3} t^{3}}^{-1}\right) .
$$

Now we have

$$
\begin{aligned}
& \Pi_{q}\left(\mathfrak{K}_{1, q, t}\right)=\mathbb{Z}\left[Y_{1, a}\left(1+A_{1, a q^{3}}^{-1}\right), Y_{2, a}^{ \pm 1}\right]_{a \in q^{\mathbb{Z}}}=\mathfrak{K}_{1, q}, \\
& \Pi_{t}\left(\mathfrak{K}_{1, q, t}\right)=\mathbb{Z}\left[Y_{1, a}\left(1+A_{i,-a t}^{-1}\right),\left(Y_{2, a} Y_{2, a \epsilon^{2}} Y_{2, a \epsilon^{4}}\right)^{ \pm 1}\right]_{a \in \epsilon^{\mathbb{Z}} \mathbb{Z}^{\mathbb{Z}}}=\mathfrak{K}_{2, t}^{L},
\end{aligned}
$$

as by Lemma 5.2 we have $Y_{1, a}\left(1+A_{1,-a t}^{-1}\right)=Z_{1, a}\left(1+B_{i, a t}^{-1}\right)$.

As for the case $r=2$, we define the analogue of $P^{\prime} \subset P$ in $\mathcal{Y}_{q}$,

$$
\mathcal{Y}_{q}^{\prime}=\mathbb{Z}\left[Y_{1, a}^{ \pm 1},\left(Y_{2, a q^{2}} Y_{2, a} Y_{2, a q^{-2}}\right)^{ \pm 1}\right]_{a \in q^{\mathbb{Z}}} .
$$




\subsection{EXAMPLES}

Now we have to check the existence of interpolating $(q, t)$-characters in some elementary cases. First, consider the following interpolating $(q, t)$-character:

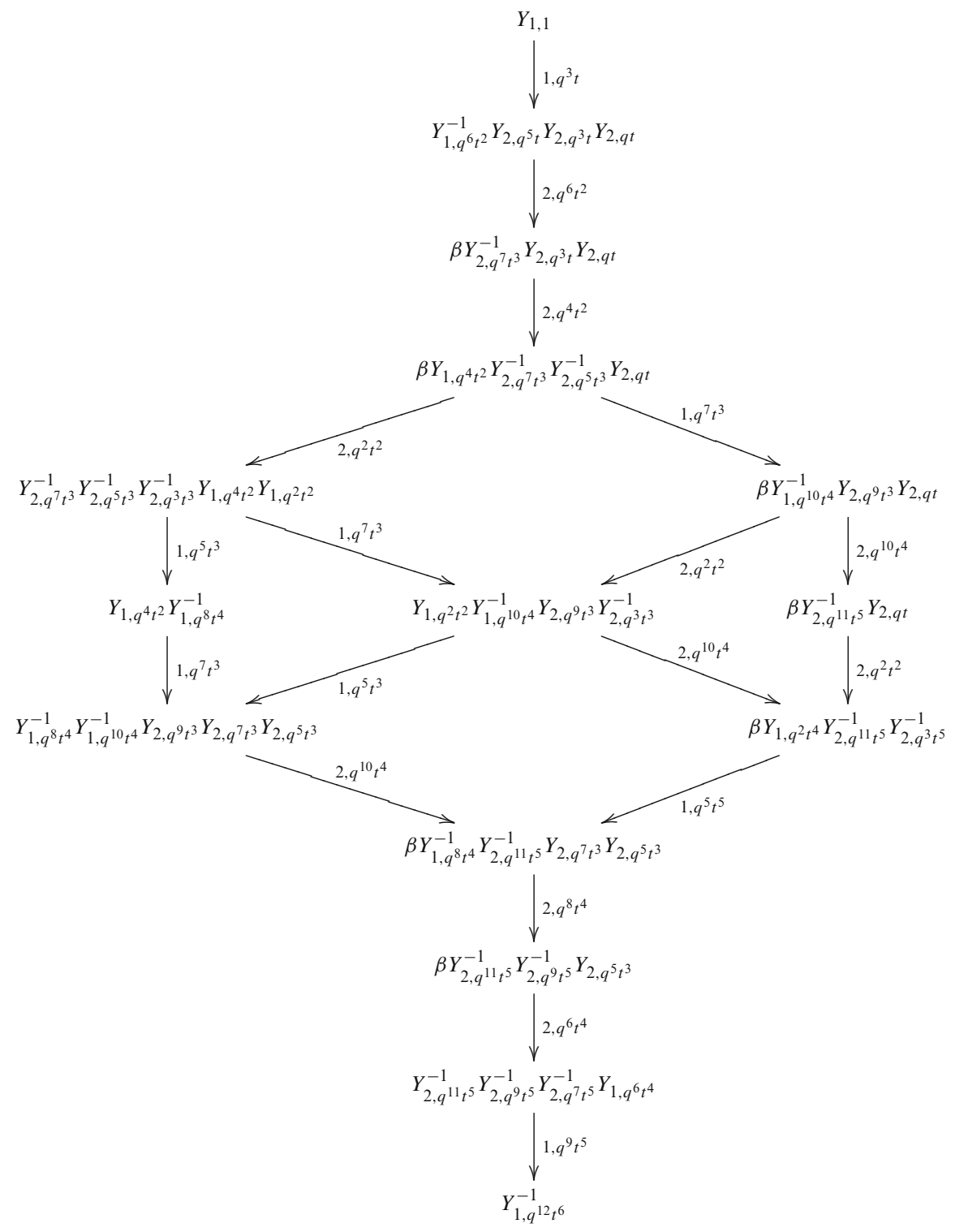

Here we have to check that it is in the $\mathfrak{K}$ as a priori it is unclear that

$$
\begin{aligned}
& \beta Y_{1, q^{10} t^{4}}^{-1} Y_{2, q^{9} t^{3}} Y_{2, q t}+Y_{1, q^{2} t^{2}} Y_{1, q^{10} t^{4}}^{-1} Y_{2, q^{9} t^{3}} Y_{2, q^{3} t^{3}}^{-1}+\beta Y_{2, q^{11} t^{5}}^{-1} Y_{2, q t} \\
& \quad+\beta Y_{1, q^{2} t^{4} Y_{2, q^{11} t^{5}} Y_{2, q^{3} t^{5}}^{-1}}
\end{aligned}
$$


is in $\mathfrak{K}_{2, q, t}$. But if we subtract $\beta Y_{2, q t}\left(1+A_{2, q^{2} t^{2}}^{-1}\right) Y_{2, q^{9} t^{3}}\left(1+A_{2, q^{10} t^{4}}^{-1}\right) Y_{1, q^{10} t^{4}}^{-1} \in \mathfrak{K}_{2, q, t}$, we get

$$
(1-\beta) Y_{2, q^{3} t^{3}}^{-1} Y_{2, q^{9} t^{3}} Y_{1, q^{10} t^{4}}^{-1} Y_{1, q^{2} t^{2}}=(1-\beta) Y_{1, q^{10} t^{4}}^{-1} Y_{1, q^{2} t^{2}} \in \mathfrak{K}_{2, q, t} .
$$

By specializing at $t=1$, we get the $q$-character of the 15-dimensional fundamental representation of $U_{q}\left(\widehat{G_{2}}\right)$ as computed in [9, Appendix]. By specializing at $q=\epsilon$, we get the following:

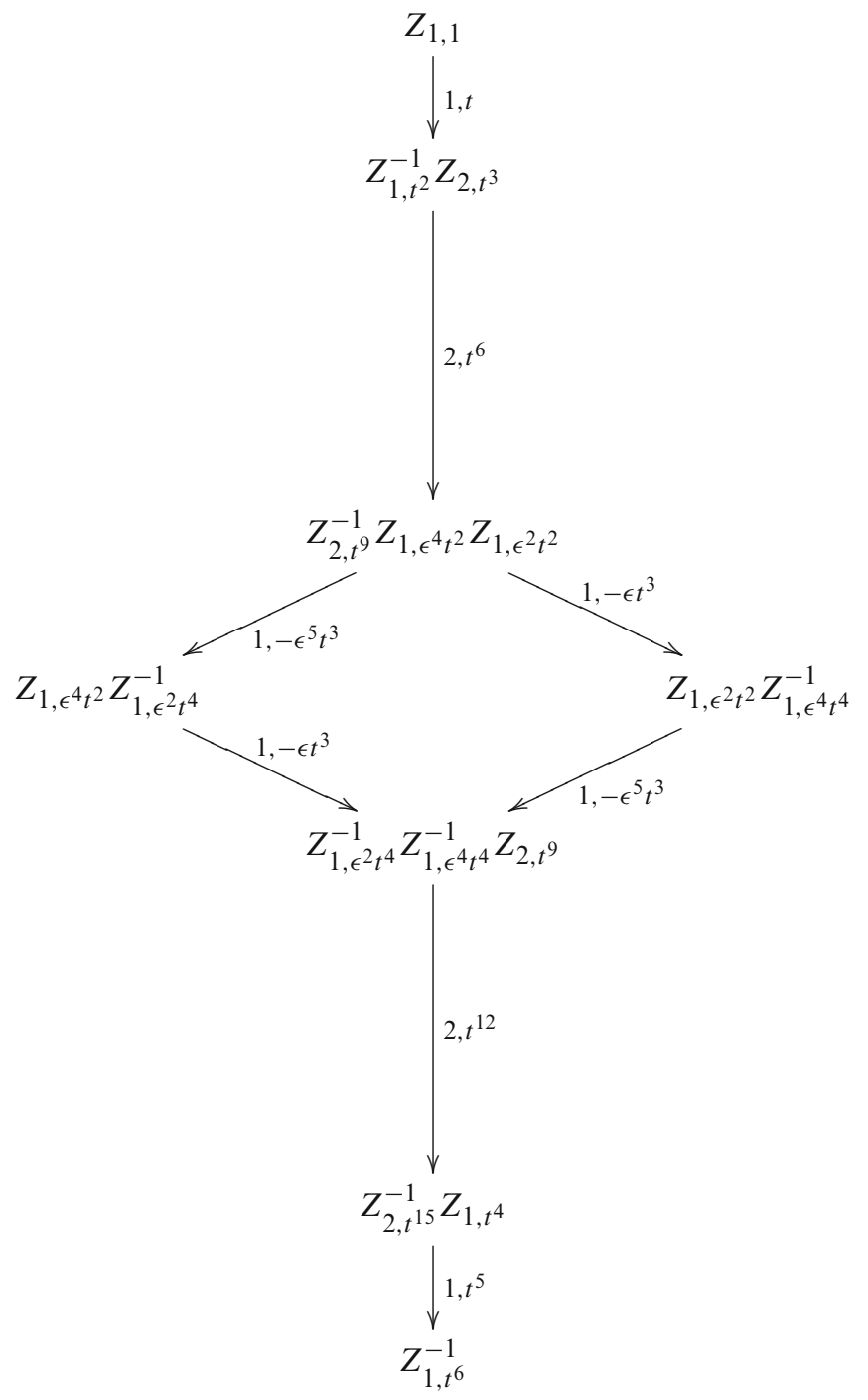

This is the twisted $t$-character of the 8-dimensional fundamental representation of $U_{t}\left(D_{4}^{(3)}\right)$ as computed in [13, Section 11.2]. 
Now we have to consider the case of the monomial $Y_{2,1} Y_{2, q^{2}} Y_{2, q^{4}}=W_{2, q^{2}}$. The dimension of the corresponding KR module of $U_{q}\left(G_{2}^{(1)}\right)$ is 133 (this can be obtained, for example, from the $T$-system proved in [11]: let $T_{k}^{(i)}$ be the dimension of a KR module of highest weight $k \omega_{i}$. Then for the fundamental representations we have $T_{1}^{(1)}=15, T_{1}^{(2)}=7$, so $T_{2}^{(2)}=\left(T_{1}^{(2)}\right)^{2}-T_{1}^{(1)}=34$ and $T_{3}^{(2)}=\left(T_{1}^{(2)}\right)^{-1}\left(\left(T_{2}^{(2)}\right)^{2}-\right.$ $\left.\left.\left(T_{1}^{(1)}\right)^{2}\right)=133\right)$.

There is also an interpolating $(q, t)$-character in this case. We do not list all 133 monomials, but we list the 29 monomials (with multiplicity) which do not have $\beta$ in their coefficient:

$$
\begin{aligned}
& 2{ }_{1} 2_{q^{2}} 2 q^{4} ; 2_{q^{2} t^{2}}^{-1} 2_{q^{4} t^{2}}^{-1} 2_{q^{6} t^{2}}^{-1} 1_{q^{5} t} 1_{q^{3} t} 1_{q t} ; 1_{q^{3} t} 1_{q t} 1_{q^{11} t^{3}}^{-1} 2_{q^{2} t^{2}}^{-1} 2_{q^{4} t^{2}}^{-1} 2_{q^{8} t^{2}} 2_{q^{10} t^{2}} ; 1_{q^{5} t} 1_{q^{9} t^{3}}^{-1} 1_{q t} 2_{q^{2} t^{2}}^{-1} 2_{q^{8} t^{2}} ; \\
& 1_{q^{5} t} 1_{q^{3} t} 1_{q^{7} t^{3}}^{-1} ; 1_{q^{9} t^{3}}^{-1} 1_{q t} 1_{q^{11} t^{3}}^{-1} 2_{q^{2} t^{2}}^{-1} 2_{q^{6} t^{2}} 2_{q^{8} t^{2}}^{2} 2_{q^{10} t^{2}} ; 1_{q^{5} t} 1_{q^{9} t^{3}}^{-1} 1_{q^{7} t^{3}}^{-1}{ }^{2} q^{4} t^{2}{ }_{q^{6} t^{2}} 2_{q^{8} t^{2}} \\
& 1_{q^{11} t^{3}}^{-1} 1_{q^{3} t} 1_{q^{7} t^{3}}^{-1} 2_{q^{10} t^{2}} 2_{q^{8} t^{2}} 2_{q^{6} t^{2}} ; 1_{q t} 1_{q^{7} t^{3}} 2_{q^{2} t^{2}}^{-1} 2_{q^{8} t^{4}}^{-1} 2_{q^{8} t^{2}} 2_{q^{10} t^{4}}^{-1} 2_{q^{12} t^{4}}^{-1} ; 1_{q^{5} t} 1_{q^{5} t^{3}} 2_{q^{6} t^{4}}^{-1} 2_{q^{8} t^{4}}^{-1} 2_{q^{10} t^{4}}^{-1} ; \\
& 1_{q^{3} t} 1_{q^{9} t^{3}} 2_{q^{12} t^{4}}^{-1} 2_{q^{10} t^{4}}^{-1} 2_{q^{8} t^{4}}^{-1} ; 1_{q^{9} t^{3}}^{-1} 1_{q^{7} t^{3}}^{-1} 1_{q^{11} t^{3}}^{-1} 2_{q^{4} t^{2}} 2_{q^{6} t^{2}}^{2} 2_{q^{8} t^{2}}^{2}{ }^{2} q^{10} t^{2} ; 1_{q t} 1_{q^{13} t^{5}}^{-1} 2_{q^{2} t^{2}}^{-1} 2_{q^{8} t^{2}} ; 1_{q^{5} t} t_{q^{11} t^{5}}^{-1} \\
& 1_{q^{3} t} 1_{q^{15} t^{5}}^{-1} 2_{q^{14} t^{4}} 2_{q^{8} t^{4}}^{-1} ; 2 \times 2 q_{q^{4} t^{2}} 2_{q^{6} t^{2}} 2_{q^{10} t^{4}}^{-1} 2_{q^{12} t^{4}}^{-1} ; 1_{q^{7} t^{3}}^{-1} 1_{q^{13} t^{5}}^{-1} q_{q^{4} t^{2}} 2_{q^{6} t^{2}} 2_{q^{8} t^{2}} \\
& 1_{q^{11} t^{3}}^{-1} 1_{q^{11} t^{5}}^{-1}{ }^{-1} q^{10} t^{2} 2 q^{8} t^{2} 2 q^{6} t^{2} ; 1_{q^{9} t^{3}}^{-1} 1_{q^{15} t^{5}}^{-1}{ }_{q^{14} t^{4}} 2_{q^{6} t^{4}} 2_{q^{4} t^{4}} ; 2_{q^{6} t^{4}}^{-1} 2_{q^{8} t^{4}}^{-2} 2_{q^{10} t^{4}}^{-2} 2_{q^{12} t^{4}}^{-1} q_{q^{5} t^{3}} 1_{q^{7} t^{3}} 1_{q^{9} t^{3}} ; \\
& 2_{q^{6} t^{4}}^{-1}{ }_{q^{8} t^{4}}^{-1}{ }_{q^{10} t^{4}}^{-1} 2 q^{14} t^{4} 1_{q^{5} t^{3}} 1_{q^{7} t^{3}} 1_{q^{15} t^{5}}^{-1} ; 2_{q^{6} t^{4}}^{-1} 2_{q^{8} t^{4}}^{-1} 2_{q^{10} t^{4}}^{-1}{ }_{q^{5} t^{3}} 1_{q^{13} t^{5}}^{-1} 1_{q^{9} t^{3}} \\
& 2_{q^{8} t^{4}}^{-1} q_{q^{10} t^{4}}^{-1} 2_{q^{12} t^{4}}^{-1}{\underset{q}{q^{11} t^{5}}}_{q^{7} t^{3}}^{-1} 1_{q^{9} t^{3}} ; 2_{q^{6} t^{4}}^{-1} 2_{q^{12} t^{4}} 2_{q^{14} t^{4}} 1_{q^{5} t^{3}} 1_{q^{13} t^{5}}^{-1} 1_{q^{15} t^{5}}^{-1} ; 1_{q^{11} t^{5}}^{-1}{\underset{q}{13} t^{5}}^{-1} 1_{q^{9} t^{3}} ; \\
& 2_{q^{8} t^{4}}^{-1} 2_{q^{14} t^{4}} 1_{q^{11} t^{5}}^{-1} 1_{q^{7} t^{3}} 1_{q^{15} t^{5}}^{-1} ; 1_{q^{11} t^{5}}^{-1} 1_{q^{13} t^{5}}^{-1}{ }_{q^{15} t^{5}}^{-1} 2_{q^{14} t^{4}} 2_{q^{12} t^{4}} 2_{q^{10} t^{4}} ; 2_{q^{16} t^{6}}^{-1} 2_{q^{14} t^{6}}^{-1} 2_{q^{12} t^{6}}^{-1} .
\end{aligned}
$$

As the other terms disappear when we specialize at $q=\epsilon$, we can compute the specialization from the above terms which is given in the figure bellow. We get the twisted $t$-character of the 29-dimensional fundamental representation of $U_{t}\left(D_{4}^{(3)}\right)$ as computed in [13, Section 11.2]. 


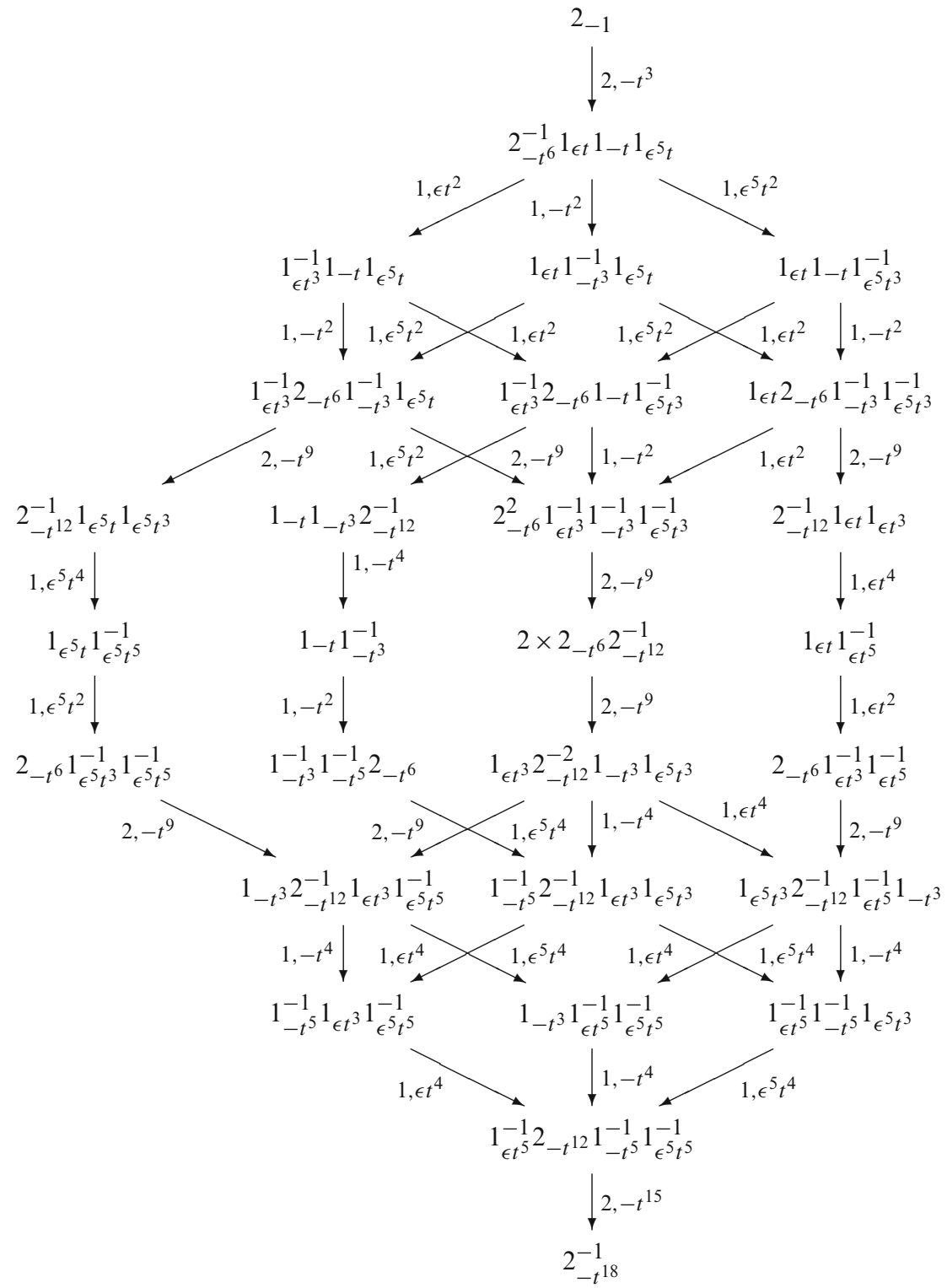

\subsection{CONCLUSION}

With the existence of the two elementary interpolating $(q, t)$-characters in the last subsection, we can conclude the proof of the two main results of this section. We define $\mathfrak{K}$ as for the case $r=2$ and we have the following:

THEOREM 5.4. For all dominant $m$ there is a unique $F(m) \in \mathfrak{K}$ such that $m$ is the unique dominant monomial of $F(m)$. 
As in the double-laced case, we have the notion of Langlands dual representation and interpolating $(q, t)$-character in $\mathfrak{K}$ with highest monomial in $\widetilde{\mathcal{Y}}_{q, t} \backslash \beta \widetilde{\mathcal{Y}}_{q, t}$. We get the following consequence of Theorem 5.4:

THEOREM 5.5. An irreducible tensor product of $K R$ modules over $U_{q}\left(G_{2}^{(1)}\right)$ of highest monomial in $\mathcal{Y}_{q}^{\prime}$ has a Langlands dual representation. Moreover, the Langlands dual representation of a KR module over $U_{q}\left(G_{2}^{(1)}\right)$ is a KR module over $U_{t}\left(D_{4}^{(3)}\right)$.

\section{From Twisted to Untwisted Types}

In this section we describe the Langlands duality in the opposite direction: from a twisted quantum algebra $U_{t}\left({ }^{L} \hat{\mathfrak{g}}\right)$ to an untwisted quantum affine algebra $U_{q}(\hat{\mathfrak{g}})$. We prove the existence of interpolating $(t, q)$-characters and we prove the duality for irreducible tensor product of KR modules (for this duality we have to use a slightly generalized definition of KR modules over twisted quantum affine algebras).

\subsection{DOUBLE-LACED CASES}

We use the notation of Section 3, in particular, for $\phi, \epsilon$. Note that $q_{i}=q^{r_{i}}$ and not $q^{r_{i}^{\vee}}$. We need the function $\alpha^{L}(t, q)$ such that $\alpha^{L}(t, \epsilon)=1$ and $\alpha^{L}(1, q)=0$ defined by $\alpha^{L}(t, q)=1-\alpha(q, t)$. Consider the ring

$$
\begin{gathered}
\mathcal{Y}_{t, q}^{L}=\mathbb{Z}\left[X_{i, a}^{ \pm 1}, \alpha^{L} z_{i, a}^{ \pm 1}, \alpha^{L}\right]_{i \in I, a \in \mathcal{C}} \subset \mathbb{Z}\left[z_{i, a}^{ \pm 1}, \alpha^{L}\right]_{i \in I, a \in \mathcal{C}}, \\
\text { where } X_{i, a}= \begin{cases}z_{i, a} & \text { if } i \in I_{2}^{\vee}=I_{1}, \\
z_{i, a q^{-1}} z_{i, a q} & \text { if } i \in I_{1}^{\vee}=I_{2} .\end{cases}
\end{gathered}
$$

We then have surjective specialization maps, respectively, at $q=\epsilon$ and $t=1$,

$$
\begin{aligned}
& \Pi_{t}^{L}: \mathcal{Y}_{t, q}^{L} \rightarrow \mathbb{Z}\left[Z_{i, a_{i}^{r_{i}}}^{ \pm 1}\right]_{i \in I, a \in \epsilon^{\mathbb{Z}} t^{\mathbb{Z}}}=\mathcal{Y}_{t}^{L}, \\
& \Pi_{q}^{L}: \mathcal{Y}_{t, q}^{L} \rightarrow \mathbb{Z}\left[Y_{i, a}^{ \pm 1}\right]_{i \in I, a \in q^{\mathbb{Z}}}=\mathcal{Y}_{q},
\end{aligned}
$$

where for $a \in \mathcal{C}, i \in I$, we assign

$$
X_{i, a} \mapsto Y_{i, a} \quad \text { and } \quad z_{i, a} \mapsto Z_{i,(-1)^{1+\phi(i)}(a)^{r_{i}^{\vee}}}
$$

Note that for $i \in I_{2}^{\vee}, a \in \epsilon^{\mathbb{Z}} t^{\mathbb{Z}}, \Pi_{t}^{L}\left(z_{i, a}\right)=\Pi_{t}^{L}\left(z_{i,-a}\right)=Z_{i, a^{2}}$. We have the ideals

$$
\begin{aligned}
\operatorname{Ker}\left(\Pi_{t}^{L}\right) & =\left\langle\left(\alpha^{L}-1\right),\left(X_{i, a q}-X_{i, a \epsilon}\right), \alpha^{L}\left(z_{i, a q}-z_{i, a \epsilon}\right),\left(z_{j, a}-z_{j,-a}\right)\right\rangle_{i \in I, j \in I_{2}^{\vee}, a \in \mathcal{C}}, \\
\operatorname{Ker}\left(\Pi_{q}^{L}\right) & =\left\langle\alpha^{L},\left(X_{i, a t}-X_{i, a}\right)\right\rangle_{i \in I, a \in \mathcal{C}} .
\end{aligned}
$$


The ideal $\operatorname{Ker}\left(\Pi_{t}^{L}\right) \cap \operatorname{Ker}\left(\Pi_{q}^{L}\right)$ is generated by the elements

$$
\begin{aligned}
& \alpha^{L}\left(\alpha^{L}-1\right), \alpha^{L}\left(z_{i, a q}-z_{i, a \epsilon}\right), \alpha^{L}\left(z_{j, a}-z_{j,-a}\right), \\
& \left(\alpha^{L}-1\right)\left(X_{i, a t}-X_{i, a}\right),\left(X_{i, a}-X_{i, a t}\right)\left(X_{k, b q}-X_{k, b \epsilon}\right),\left(X_{i, a}-X_{i, a t}\right)\left(z_{j, b}-z_{j,-b}\right),
\end{aligned}
$$

for $i, k \in I, j \in I_{2}^{\vee}, a, b \in \mathcal{C}$. We will work in the ring

$$
\tilde{\mathcal{Y}}_{t, q}^{L}=\mathcal{Y}_{t, q}^{L} /\left(\operatorname{Ker}\left(\Pi_{t}^{L}\right) \cap \operatorname{Ker}\left(\Pi_{q}^{L}\right)\right)
$$

We use the notion of monomial, dominant monomial as above.

DEFINITION 6.1. For $i \in I$ and $a \in \mathcal{C}$ we define

$$
\widetilde{B}_{i, a}=z_{i, a\left(q_{i} t\right)^{-1} z_{i, a q_{i}}+} \times \prod_{j \in I, C_{j, i}=-1} z_{j, a}^{-1} \times \prod_{j \in I, C_{j, i}=-2} z_{j, a q^{-1}}^{-1} z_{j, a q}^{-1} .
$$

LEMMA 6.2. We have $\Pi_{t}^{L}\left(\widetilde{B}_{i, a}\right)=B_{i,\left(\Pi_{t}^{L}(a)\right)^{r} r_{i}^{\vee}(-1)^{\phi(i)}}$ for $i \in I, a \in \mathcal{C}$.

We have $\Pi_{q}^{L}\left(\widetilde{B}_{i, a q^{-1}} \widetilde{B}_{i, a q}\right)=A_{i, \Pi_{q}^{L}(a)}$ for $i \in I_{1}^{\vee}, a \in \mathcal{C}$.

We have $\Pi_{q}^{L}\left(\widetilde{B}_{i, a}\right)=A_{i, \Pi_{q}^{L}(a)}$ for $i \in I_{2}^{\vee}$, a $\in \mathcal{C}$.

Proof. Let $a^{\prime}=\Pi_{t}^{L}(a)$. For $i \in I_{1}^{\vee}$, we have

$$
\begin{aligned}
\Pi_{t}^{L}\left(\widetilde{B}_{i, a}\right)= & z_{i,-a^{\prime} t^{-1}} z_{i,-a^{\prime} t} \times \prod_{j \in I, C_{j, i}=-1} z_{j, a^{\prime}}^{-1} \\
= & Z_{i,(-1)^{\phi(i)} a^{\prime} t^{-1}} Z_{i,(-1)^{\phi(i)} a^{\prime} t} \times \prod_{j \in I_{1}^{\vee}, C_{j, i}=-1} Z_{j,(-1)^{\phi(i)} a^{\prime}}^{-1} \\
& \times \prod_{j \in I_{2}^{\vee}, C_{j, i}=-1} Z_{j,\left(a^{\prime}\right)^{2}}^{-1},
\end{aligned}
$$

which is equal to $B_{i, a^{\prime}(-1)^{\phi(i)}}$. Indeed if there is $j \in I_{2}^{\vee}=I_{1}$ such that $C_{j, i}=-1$, we have $\phi(j)=1$ and so $Z_{j,\left(a^{\prime}\right)^{2}}=z_{j, a^{\prime}}$.

For $i \in I_{2}^{\vee}$, we have

$$
\begin{aligned}
& \Pi_{t}^{L}\left(\widetilde{B}_{i, a}\right)=z_{i,-\epsilon a^{\prime} t^{-1}} z_{i, \epsilon a^{\prime} t} \times \prod_{j \in I, C_{j, i}=-1} z_{j, a^{\prime}}^{-1} \times \prod_{j \in I, C_{j, i}=-2} z_{j,-\epsilon a^{\prime}}^{-1} z_{j, \epsilon a^{\prime}}^{-1} \\
& =Z_{i,(-1)^{\phi(i)}\left(a^{\prime}\right)^{2} t^{-2}} Z_{i,(-1)^{\phi(i)}\left(a^{\prime}\right)^{2} t^{2}} \times \prod_{j \in I, C_{j, i}=-1} Z_{j,(-1)^{\phi(i)}\left(a^{\prime}\right)^{2}}^{-1} \\
& \times \prod_{j \in I, C_{j, i}=-2} Z_{j, \epsilon a^{\prime}}^{-1} Z_{j,-\epsilon a^{\prime}}^{-1}
\end{aligned}
$$


which is equal to $B_{i,(-1)^{\phi(i)}\left(a^{\prime}\right)^{2}}$. Indeed, if there is $j \in I$ such that $C_{j, i}=-2$, we have $\phi(i)=1$ and so $\left(\epsilon a^{\prime}\right)^{2}=-\left(a^{\prime}\right)^{2}=(-1)^{\phi(i)}\left(a^{\prime}\right)^{2}$.

Let $a^{\prime \prime}=\Pi_{q}^{L}(a)$. For $i \in I_{1}^{\vee}$, we have

$$
\begin{aligned}
& \Pi_{q}^{L}\left(\left(\widetilde{B}_{i, a q^{-1}} \widetilde{B}_{i, a q}\right)\right)=\left(z_{i, a^{\prime \prime} q^{-3}} z_{i, a^{\prime \prime} q^{-1}}\right)\left(z_{i, a^{\prime \prime} q} z_{i, a^{\prime \prime} q^{3}}\right) \times \prod_{j \in I, C_{j, i}=-1} z_{j, a^{\prime \prime} q^{-1}}^{-1} z_{j, a^{\prime \prime} q}^{-1} \\
& =Y_{i, a^{\prime \prime} q^{-2}} Y_{i, a^{\prime \prime} q^{2}} \times \prod_{j \in I_{1}^{\vee}, C_{j, i}=-1} Y_{j, a^{\prime \prime}}^{-1} \\
& \times \prod_{j \in I_{2}^{\vee}, C_{j, i}=-1} Y_{j, a^{\prime \prime} q^{-1}}^{-1} Y_{j, a^{\prime \prime} q}^{-1}=A_{i, a^{\prime \prime}}
\end{aligned}
$$

For $i \in I_{2}^{\vee}$, we have

$$
\begin{aligned}
\Pi_{q}^{L}\left(\widetilde{B}_{i, a}\right) & =z_{i, a^{\prime \prime} q^{-1}} z_{i, a^{\prime \prime} q} \times \prod_{j \in I, C_{j, i}=-1} z_{j, a^{\prime \prime}}^{-1} \times \prod_{j \in I, C_{j, i}=-2}\left(z_{\left.j, a^{\prime \prime} q^{-1} z_{j, a^{\prime \prime} q}\right)^{-1}}\right. \\
& =Y_{i, a^{\prime \prime} q^{-1}} Y_{i, a^{\prime \prime} q} \times \prod_{j \in I, C_{j, i}<0} Y_{j, a^{\prime \prime}}^{-1}=A_{i, a^{\prime \prime}}
\end{aligned}
$$

For $i \in I_{1}^{\vee}$, consider the subalgebra $\mathfrak{K}_{i, t, q}^{L}$ of $\mathcal{Y}_{t, q}^{L}$ equal to

$$
\mathbb{Z}\left[X_{i, a}\left(1+\alpha^{L} \widetilde{B}_{i, a q^{3} t}^{-1}+\alpha^{L} \widetilde{B}_{i, a q t}^{-1}+\widetilde{B}_{i, a q^{3} t}^{-1} \widetilde{B}_{i, a q t}^{-1}\right), \alpha^{L} z_{i, a}\left(1+\widetilde{B}_{i, a q^{2} t}^{-1}\right), X_{j, a}^{ \pm 1}, \alpha^{L} z_{j, a}^{ \pm 1}, \alpha^{L}\right]_{a \in \mathcal{C}, j \neq i},
$$

and for $i \in I_{2}^{L}$,

$$
\mathfrak{K}_{i, t, q}^{L}=\mathbb{Z}\left[z_{i, a}\left(1+\widetilde{B}_{i, a q t}^{-1}\right), X_{j, a}^{ \pm 1}, \alpha^{L} z_{j, a}^{ \pm 1}, \alpha^{L}\right]_{a \in \mathcal{C}, j \neq i}
$$

Then we have the following:

LEMMA 6.3. We have $\Pi_{t}^{L}\left(\mathfrak{K}_{i, t, q}^{L}\right)=\mathfrak{K}_{i, t}^{L}$ and $\Pi_{q}^{L}\left(\mathfrak{K}_{i, t, q}^{L}\right)=\mathfrak{K}_{i, q}$ for $i \in I$.

Proof. For $i \in I_{1}^{\vee}, \Pi_{t}^{L}\left(\mathfrak{K}_{i, t, q}^{L}\right)$ is equal to

$$
\begin{aligned}
& \mathbb{Z}\left[z_{i,-\epsilon a} z_{i, \epsilon a}\left(1+B_{i,-\epsilon a t(-1)^{\phi(i)}}^{-1}\right)\left(1+B_{i, \epsilon a t(-1)^{\phi(i)}}^{-1}\right), z_{i, a}\left(1+B_{i, a t(-1)^{\phi(i)+1}}^{-1}\right), z_{j, a}^{ \pm 1}\right]_{a \in t^{\mathbb{Z}} \epsilon^{\mathbb{Z}}, j \neq i} \\
& =\mathbb{Z}\left[Z_{i, a(-1)^{\phi(i)+1}}\left(1+B_{i, a(-1)^{\phi(i)+1} t}^{-1}\right), Z_{j, a^{r}}^{ \pm 1}\right]_{a \in t^{\mathbb{Z}} \epsilon^{\mathbb{Z}}, j \neq i}=\mathfrak{K}_{i, t}^{L} .
\end{aligned}
$$


We also have

$$
\begin{aligned}
\Pi_{q}^{L}\left(\mathfrak{K}_{i, t, q}^{L}\right) & =\mathbb{Z}\left[\Pi_{q}^{L}\left(X_{i, a}\left(1+\widetilde{B}_{i, a q^{3} t}^{-1} \widetilde{B}_{i, a q t}^{-1}\right)\right), \Pi_{q}^{L}\left(X_{j, a}^{ \pm 1}\right)\right]_{a \in \mathcal{C}, j \neq i} \\
& =\mathbb{Z}\left[Y_{i, a}\left(1+A_{i, a q^{2}}^{-1}\right), Y_{j, a}^{ \pm 1}\right]_{a \in q^{\mathbb{Z}}, j \neq i}=\mathfrak{K}_{i, q} .
\end{aligned}
$$

Now for $i \in I_{2}^{\vee}$, we have

$$
\begin{aligned}
\Pi_{t}^{L}\left(\mathfrak{K}_{i, t, q}^{L}\right) & =\mathbb{Z}\left[z_{i, a}\left(1+B_{i, a^{2} t^{2}(-1)^{\phi(i)+1}}^{-1}\right), z_{j, a}^{ \pm 1}\right]_{a \in t^{\mathbb{Z}} \epsilon^{\mathbb{Z}}, j \neq i} \\
& =\mathbb{Z}\left[Z_{i, a^{2}(-1)^{\phi(i)+1}}\left(1+B_{i, a^{2} t^{2}(-1)^{\phi(i)+1}}^{-1}\right), Z_{j, a^{r}}^{ \pm 1}\right]_{a \in t^{\mathbb{Z}} \epsilon^{\mathbb{Z}}, j \neq i}=\mathfrak{K}_{i, t}^{L}, \\
\Pi_{q}^{L}\left(\mathfrak{K}_{i, t, q}^{L}\right) & =\mathbb{Z}\left[Y_{i, a}\left(1+A_{i, a q}^{-1}\right), Y_{j, a}^{ \pm 1}\right]_{a \in q^{\mathbb{Z}, j \neq i}}=\mathfrak{K}_{i, q} .
\end{aligned}
$$

We set

$$
\left(\mathcal{Y}_{t}^{L}\right)^{\prime}=\mathbb{Z}\left[Z_{i, a}^{ \pm 1}\right]_{i \in I_{2}^{\vee}, a \in\left(\epsilon^{\mathbb{Z}} t^{\mathbb{Z}}\right)^{2}} \otimes \mathbb{Z}\left[\left(Z_{i, a} Z_{i,-a}\right)^{ \pm 1}\right]_{i \in I_{1}^{\vee}, a \in \epsilon^{\mathbb{Z}} t^{\mathbb{Z}}} \subset \mathcal{Y}_{t}^{L}
$$

and we define $\mathfrak{K}^{L} \subset \tilde{\mathcal{Y}}_{t, q}^{L}$ as above.

As in the previous sections, we check the existence of various elements in $\mathfrak{K}^{L}$ that we call interpolating $(t, q)$-characters.

First, we suppose that $U_{t}\left({ }^{L} \widehat{\mathfrak{g}}\right)$ is of type $A_{3}^{(2)}$, and so that $U_{q}(\widehat{\mathfrak{g}})$ is of type $C_{2}^{(1)}$, with $r_{1}=r_{2}^{\vee}=2$ and $r_{2}=r_{1}^{\vee}=1$. We have $\phi(1)=0, \phi(2)=1$. We have the following interpolating $(q, t)$-character:
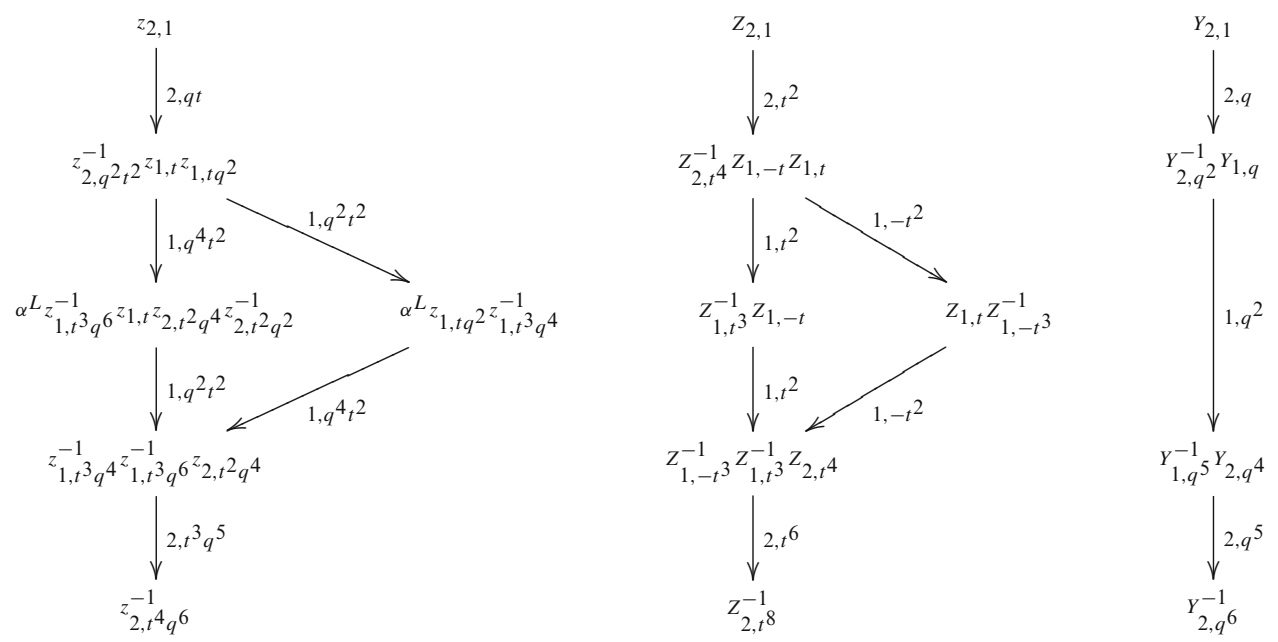
$\Pi_{t}^{L}$ gives the twisted $t$-character of a 6-dimensional fundamental representation of $U_{t}\left(A_{3}^{(2)}\right)$ and $\Pi_{q}^{L}$ the $q$-character of a 4-dimensional fundamental representation of $U_{q}\left(C_{2}^{(1)}\right)$.

We also have the following interpolating $(t, q)$-character

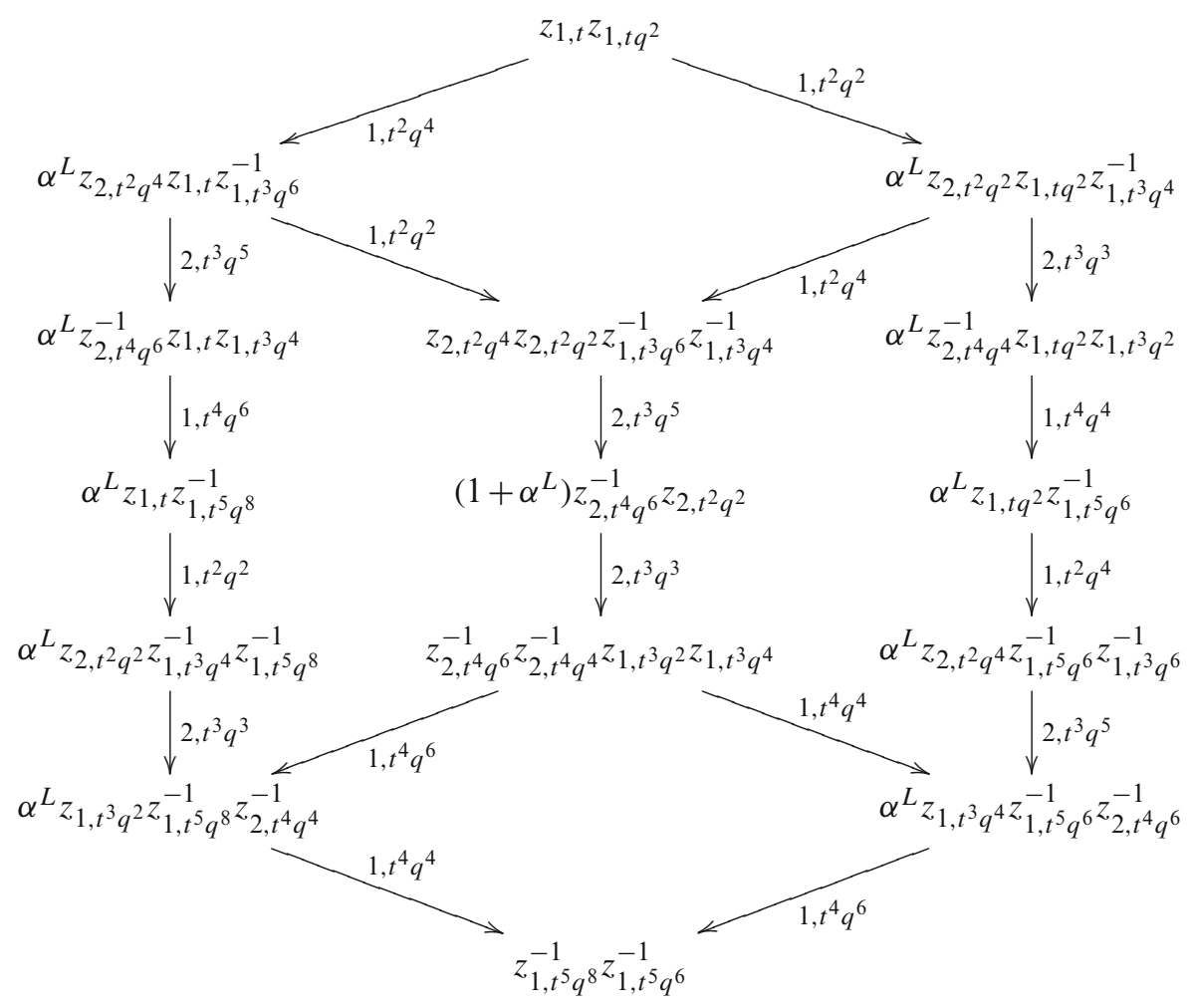

It is easy to check that it is in the $\mathfrak{K}^{L}$, for example,

$$
\begin{aligned}
& z_{2, t^{2} q^{4}} z_{2, t^{2} q^{2}} z_{1, t^{3} q^{6}}^{-1} z_{1, t^{3} q^{4}}^{-1}+\left(1+\alpha^{L}\right) z_{2, t^{4} q^{6}}^{-1} z_{2, t^{2} q^{2}}+z_{2, t^{4} q^{6}}^{-1} z_{2, t^{4} q^{4}}^{-1} z_{1, t^{3} q^{2}} z_{1, t^{3} q^{4}}
\end{aligned}
$$

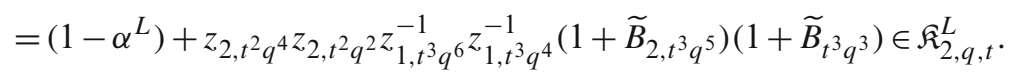

Note that the coefficients $\alpha^{L}$ are imposed by the condition that the interpolating $(t, q)$-character is in $\mathfrak{K}^{L}$, in particular the coefficient $\left(1+\alpha^{L}\right)$ of $z_{2, t^{4} q^{6}}^{-1} z_{2, t^{2} q^{2}}$.

$\Pi_{t}^{L}$ gives the twisted $t$-character of a tensor product of two 4-dimensional fundamental representation of $U_{t}\left(A_{3}^{(2)}\right)$ and $\Pi_{q}^{L}$ the $q$-character of a 5 -dimensional fundamental representation of $U_{q}\left(C_{2}^{(1)}\right)$. 


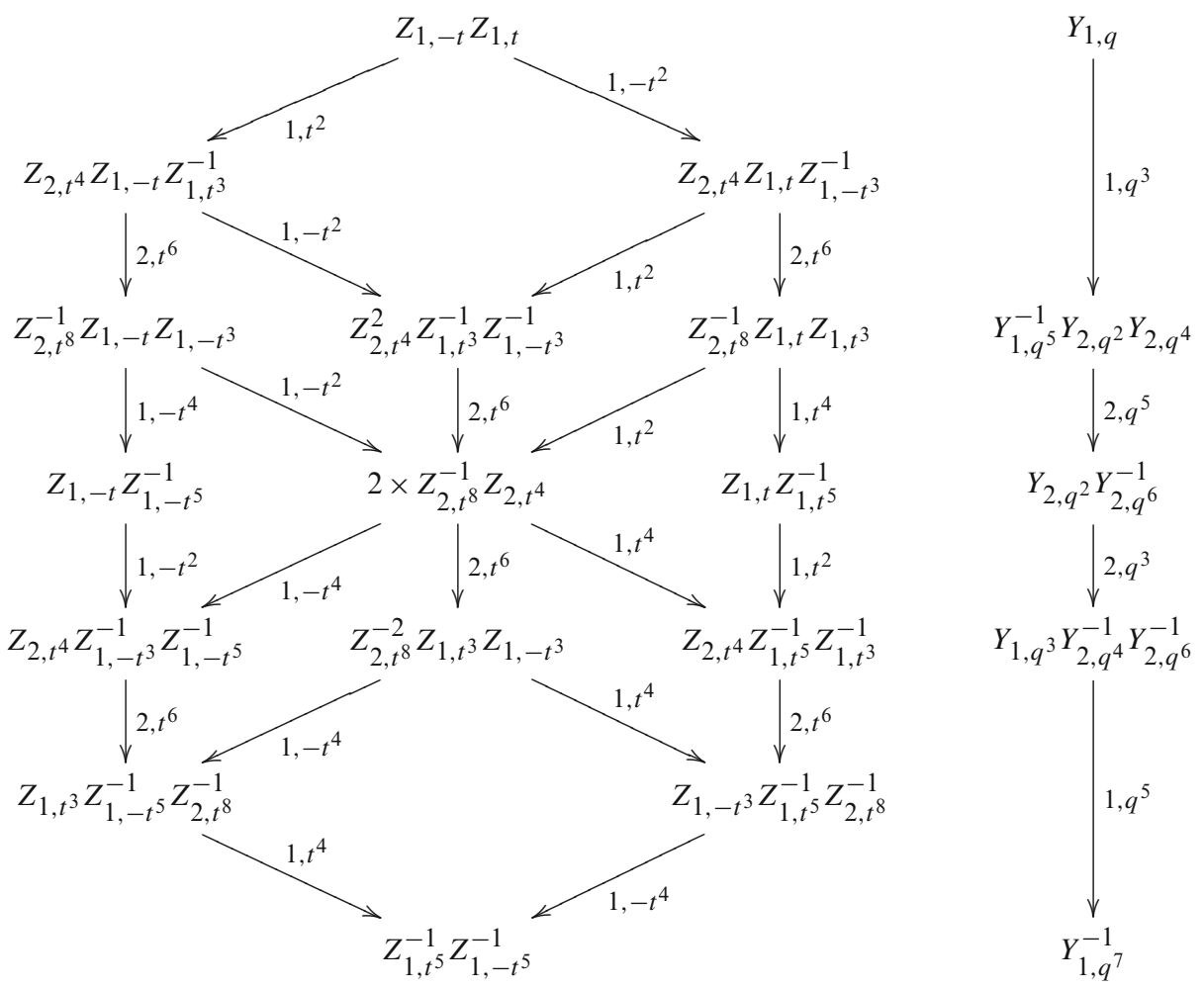

The multiplicity 2 of $Z_{2, t^{4}} Z_{2, t^{8}}^{-1}$ in the image by $\Pi_{t}^{L}$ is ramified into $1+\alpha^{L}$ in the interpolating $(t, q)$-character. That is why we get just a multiplicity 1 for $Y_{2, q^{2}} Y_{2, q^{6}}^{-1}$. Note that in particular the interpolating $(t, q)$-character can not be factorized.

Next, consider $C_{i, j}=C_{j, i}=-1$ with $r_{1}^{\vee}=r_{2}^{\vee}=1$. We choose $\phi(1)=0, \phi(2)=1$, and we have the following interpolating $(t, q)$-character.

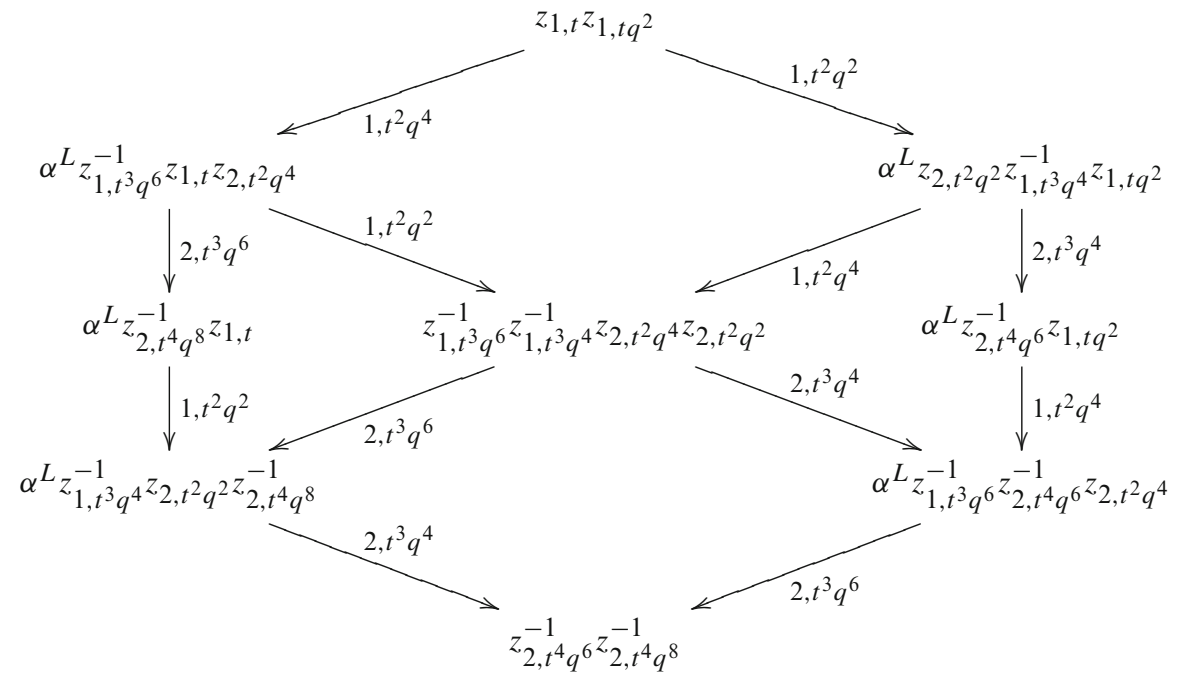


$\Pi_{t}^{L}$ gives the $t$-character of a tensor product of two 3-dimensional fundamental representation of $U_{t}\left(A_{3}^{(1)}\right)$ and $\Pi_{q}^{L}$ the $q$-character of a 3-dimensional fundamental representation of $U_{q}\left(A_{3}^{(1)}\right)$.
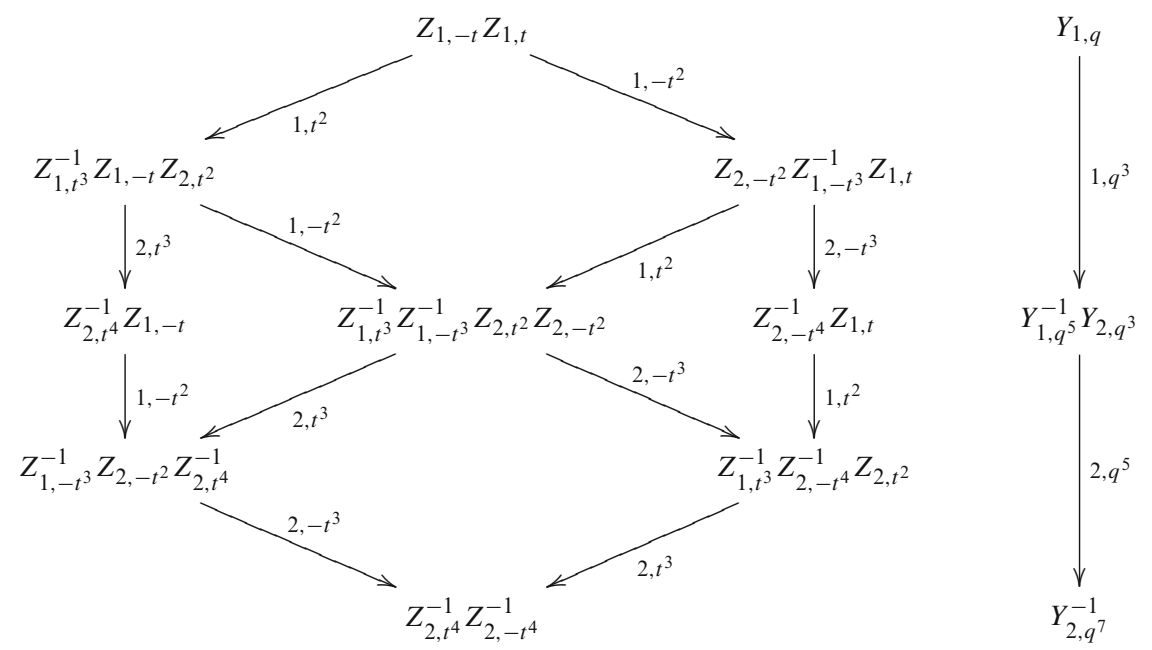

Consider $C_{i, j}=C_{j, i}=-1$ with $r_{1}^{\vee}=r_{2}^{\vee}=2$. We choose $\phi(1)=0, \phi(2)=1$. We have the following interpolating $(t, q)$-character:

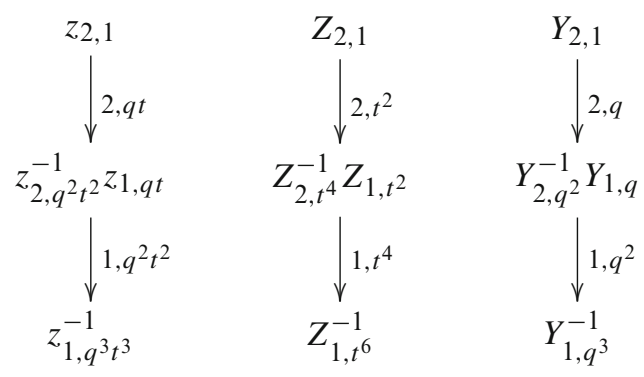

$\Pi_{t}^{L}$ gives the $t$-character of a 3-dimensional fundamental representation of $U_{t}\left(A_{3}^{(1)}\right)$ and $\Pi_{q}^{L}$ the $q$-character of a 3-dimensional fundamental representation of $U_{q}\left(A_{3}^{(1)}\right)$.

\subsection{TRIPLE-LACED CASE}

We use the notations of Section 5. We need the function $\beta^{L}(t, q)$ such that $\beta^{L}(t, \epsilon)=1$ and $\beta^{L}(1, q)=0$ defined by $\beta^{L}(t, q)=1-\beta(q, t)$.

Consider the ring

$$
\begin{gathered}
\mathcal{Y}_{t, q}^{L}=\mathbb{Z}\left[X_{i, a}^{ \pm 1}, \beta^{L} z_{i, a}^{ \pm 1}, \beta^{L}\right]_{i \in I, a \in \mathcal{C}} \subset \mathbb{Z}\left[z_{i, a}^{ \pm 1}, \beta^{L}\right]_{i \in I, a \in \mathcal{C}}, \\
\text { where } X_{i, a}= \begin{cases}z_{i, a} & \text { if } i=2, \\
z_{i, a q^{-2}} z_{i, a} z_{i, a q^{2}} & \text { if } i=1 .\end{cases}
\end{gathered}
$$


We then have the surjective specialization maps, $\Pi_{t}^{L}, \Pi_{q}^{L}$, where for $a \in \mathcal{C}$, we use

$$
X_{1, a} \mapsto Y_{1, a}, z_{1, a} \mapsto Z_{1,-a}, X_{2, a} \mapsto Y_{2, a}, z_{2, a} \mapsto Z_{2, a^{3}}
$$

Note that the identification is not one to one as $Z_{2, a^{3}}$ is identified with $z_{2, a}, z_{2, \epsilon^{2} a}$ and $Z_{2, \epsilon^{4} a}$. Note also that the identification is not involutive with respect to the identification in Section 5 as $z_{1, a}$ is identified with $Z_{1,-a}$ and note with $Z_{1, a}$.

The ideal $\operatorname{Ker}\left(\Pi_{t}^{L}\right) \cap \operatorname{Ker}\left(\Pi_{q}^{L}\right)$ is generated by the elements

$$
\begin{aligned}
& \beta^{L}\left(\beta^{L}-1\right), \beta^{L}\left(z_{i, a q}-z_{i, a \epsilon}\right), \beta^{L}\left(z_{2, a}-z_{2, a \epsilon^{2}}\right),\left(\beta^{L}-1\right)\left(X_{i, a t}-X_{i, a}\right), \\
& \left(X_{i, a}-X_{i, a t}\right)\left(X_{j, b q}-X_{j, b \epsilon}\right),\left(X_{i, a}-X_{i, a t}\right)\left(z_{2, b}-z_{2, b \epsilon^{2}}\right),
\end{aligned}
$$

for $i, j \in I, a, b \in \mathcal{C}$.

DEFINITION 6.4. For $a \in \mathcal{C}$ we define

$$
\widetilde{B}_{1, a}=z_{i, a\left(t q^{3}\right)^{-1}} z_{i, a t q^{3}} z_{2, a}^{-1}, \quad \widetilde{B}_{2, a}=z_{2, a(q t)^{-1} z_{2, a q t} z_{1, a q^{-2}}^{-1} z_{1, a}^{-1} z_{1, a q^{2}}^{-1}}
$$

LEMMA 6.5. Let $a \in \mathcal{C}$. We have

$$
\begin{aligned}
& \Pi_{t}^{L}\left(\widetilde{B}_{1, a}\right)=B_{1,\left(\Pi_{t}^{L}(a)\right)}, \Pi_{t}^{L}\left(\widetilde{B}_{2, a}\right)=B_{2,-\left(\Pi_{t}^{L}(a)\right)^{3}} \\
& \Pi_{q}^{L}\left(\widetilde{B}_{1, a q^{-2}} \widetilde{B}_{1, a} \widetilde{B}_{1, a q^{2}}\right)=A_{1,\left(\Pi_{q}^{L}(a)\right)}, \Pi_{q}^{L}\left(\widetilde{B}_{2, a}\right)=A_{2, \Pi_{q}^{L}(a)} .
\end{aligned}
$$

Proof. Let $a^{\prime}=\Pi_{t}^{L}(a)$. We have

$$
\begin{aligned}
& \Pi_{t}^{L}\left(\widetilde{B}_{1, a}\right)=z_{1,-a^{\prime} t^{-1}} z_{1,-a^{\prime} t} z_{2, a^{\prime}}^{-1}=Z_{1, a^{\prime} t^{-1}} Z_{1, a^{\prime} t} Z_{2,\left(a^{\prime}\right)^{3}}^{-1}=B_{1, a^{\prime}},
\end{aligned}
$$

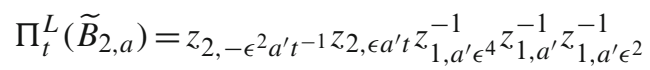

$$
\begin{aligned}
& =Z_{2,-\left(a^{\prime}\right)^{3} t^{-3}} Z_{2,-\left(a^{\prime}\right)^{3} t^{3}} Z_{1,-a^{\prime} \epsilon^{4}}^{-1} Z_{1,-a^{\prime}}^{-1} Z_{1,-a^{\prime} \epsilon^{2}}^{-1}=B_{2,-\left(a^{\prime}\right)^{3}} .
\end{aligned}
$$

Let $a^{\prime \prime}=\Pi_{q}^{L}(a)$. We have

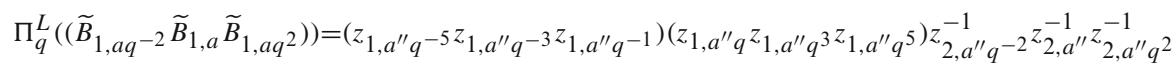

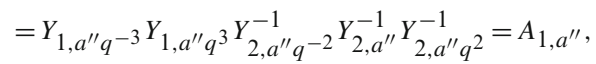

$$
\begin{aligned}
& \Pi_{q}^{L}\left(\widetilde{B}_{2, a}\right)=z_{2, a^{\prime \prime} q^{-1}} z_{2, a^{\prime \prime} q} z_{1, a^{\prime \prime}}^{-1}=Y_{2, a^{\prime \prime} q^{-1}} Y_{2, a^{\prime \prime}} Y_{1, a^{\prime \prime}}^{-1}=A_{2, a^{\prime \prime}} .
\end{aligned}
$$

Consider the subalgebra $\mathfrak{K}_{1, t, q}^{L}$ of $\mathcal{Y}_{t, q}^{L}$ generated by the

$$
\begin{aligned}
& X_{1, a}\left(1+\beta^{L} \widetilde{B}_{1, q^{5} t}^{-1}\right)\left(1+\beta^{L} \widetilde{B}_{1, q^{3} t}^{-1}\right)\left(1+\beta^{L} \widetilde{B}_{1, q t}^{-1}\right)+\left(1-\beta^{L}\right) X_{1, a} \widetilde{B}_{1, a q^{5} t}^{-1} \widetilde{B}_{1, a q^{3} t}^{-1} \widetilde{B}_{1, a q t}^{-1}, \\
& \quad \beta^{L} z_{1, a}\left(1+\widetilde{B}_{1, a q^{3} t}^{-1}\right), z_{2, a}^{ \pm 1}, \beta^{L},
\end{aligned}
$$


for $a \in \mathcal{C}$, and the subalgebra

$$
\mathfrak{K}_{2, t, q}^{L}=\mathbb{Z}\left[z_{2, a}\left(1+\widetilde{B}_{2, a q t}^{-1}\right), X_{1, a}^{ \pm 1}, \beta^{L} z_{1, a}^{ \pm 1}, \beta^{L}\right]_{a \in \mathcal{C}}
$$

Then we have the following:

LEMMA 6.6. For $i \in I$, we have $\Pi_{t}^{L}\left(\mathfrak{K}_{i, t, q}^{L}\right)=\mathfrak{K}_{i, t}^{L}$ and $\Pi_{q}^{L}\left(\mathfrak{K}_{i, t, q}^{L}\right)=\mathfrak{K}_{i, q}$.

Proof. $\Pi_{t}^{L}\left(\mathfrak{K}_{1, t, q}^{L}\right)$ is equal to

$$
\begin{aligned}
& \mathbb{Z}\left[z_{1, \epsilon^{-2} a} z_{1, a} z_{1, a \epsilon^{2}}\left(1+B_{1,-\epsilon^{2} a t}^{-1}\right)\left(1+B_{1,-a t}^{-1}\right)\left(1+B_{1,-\epsilon^{-2} a t}^{-1}\right), z_{1, a}\left(1+B_{1,-a t}^{-1}\right), z_{2, a}^{ \pm 1}\right]_{a \in t^{\mathbb{Z}} \epsilon^{\mathbb{Z}}} \\
& \quad=\mathbb{Z}\left[Z_{1,-a}\left(1+B_{1,-a t}^{-1}\right), Z_{2, a^{3}}^{ \pm 1}\right]_{a \in t^{\mathbb{Z}} \epsilon^{\mathbb{Z}}}=\mathfrak{K}_{1, t}^{L} .
\end{aligned}
$$

We also have

$$
\begin{aligned}
\Pi_{q}^{L}\left(\mathfrak{K}_{1, t, q}^{L}\right) & =\mathbb{Z}\left[\Pi_{q}^{L}\left(X_{1, a}\left(1+\widetilde{B}_{i, a q^{5} t}^{-1} \widetilde{B}_{i, a q^{3} t}^{-1} \widetilde{B}_{i, a q t}^{-1}\right)\right), \Pi_{q}^{L}\left(X_{2, a}^{ \pm 1}\right)\right]_{a \in \mathcal{C}} \\
& =\mathbb{Z}\left[Y_{1, a}\left(1+A_{1, a q^{3}}^{-1}\right), Y_{2, a}^{ \pm 1}\right]_{a \in q^{\mathbb{Z}}}=\mathfrak{K}_{1, q}, \\
\Pi_{t}^{L}\left(\mathfrak{K}_{2, t, q}^{L}\right) & =\mathbb{Z}\left[z_{2, a}\left(1+B_{2, a^{3} t^{3}}^{-1}\right), z_{1, a}^{ \pm 1}\right]_{a \in t^{\mathbb{Z}} \epsilon^{\mathbb{Z}}}=\mathbb{Z}\left[Z_{2, a^{3}}\left(1+B_{2, a^{3} t^{3}}^{-1}\right), Z_{1, a}^{ \pm 1}\right]_{a \in t^{\mathbb{Z}} \epsilon^{\mathbb{Z}}} \\
& =\mathfrak{K}_{2, t}^{L},
\end{aligned}
$$

and we have

$$
\Pi_{q}^{L}\left(\mathfrak{K}_{2, t, q}^{L}\right)=\mathbb{Z}\left[Y_{2, a}\left(1+A_{2, a q}^{-1}\right), Y_{1, a}^{ \pm 1}\right]_{a \in q^{\mathbb{Z}}}=\mathfrak{K}_{2, q}
$$

We set

$$
\left(\mathcal{Y}_{t}^{L}\right)^{\prime}=\mathbb{Z}\left[Z_{2, a}^{ \pm 1}\right]_{a \in\left(\epsilon^{\mathbb{Z}} t^{\mathbb{Z}}\right)^{3}} \otimes \mathbb{Z}\left[\left(Z_{1, a} Z_{1, a \epsilon^{2}} Z_{1, a \epsilon^{4}}\right)^{ \pm 1}\right]_{a \in \epsilon^{\mathbb{Z}} t^{\mathbb{Z}}} \subset \mathcal{Y}_{t}^{L}
$$

and we define $\mathfrak{K}^{L} \subset \tilde{\mathcal{Y}}_{t, q}^{L}$ as above.

As in the previous sections, we check the existence of elements in $\mathfrak{K}^{L}$ that we call interpolating $(t, q)$-characters. First, we have the following: 


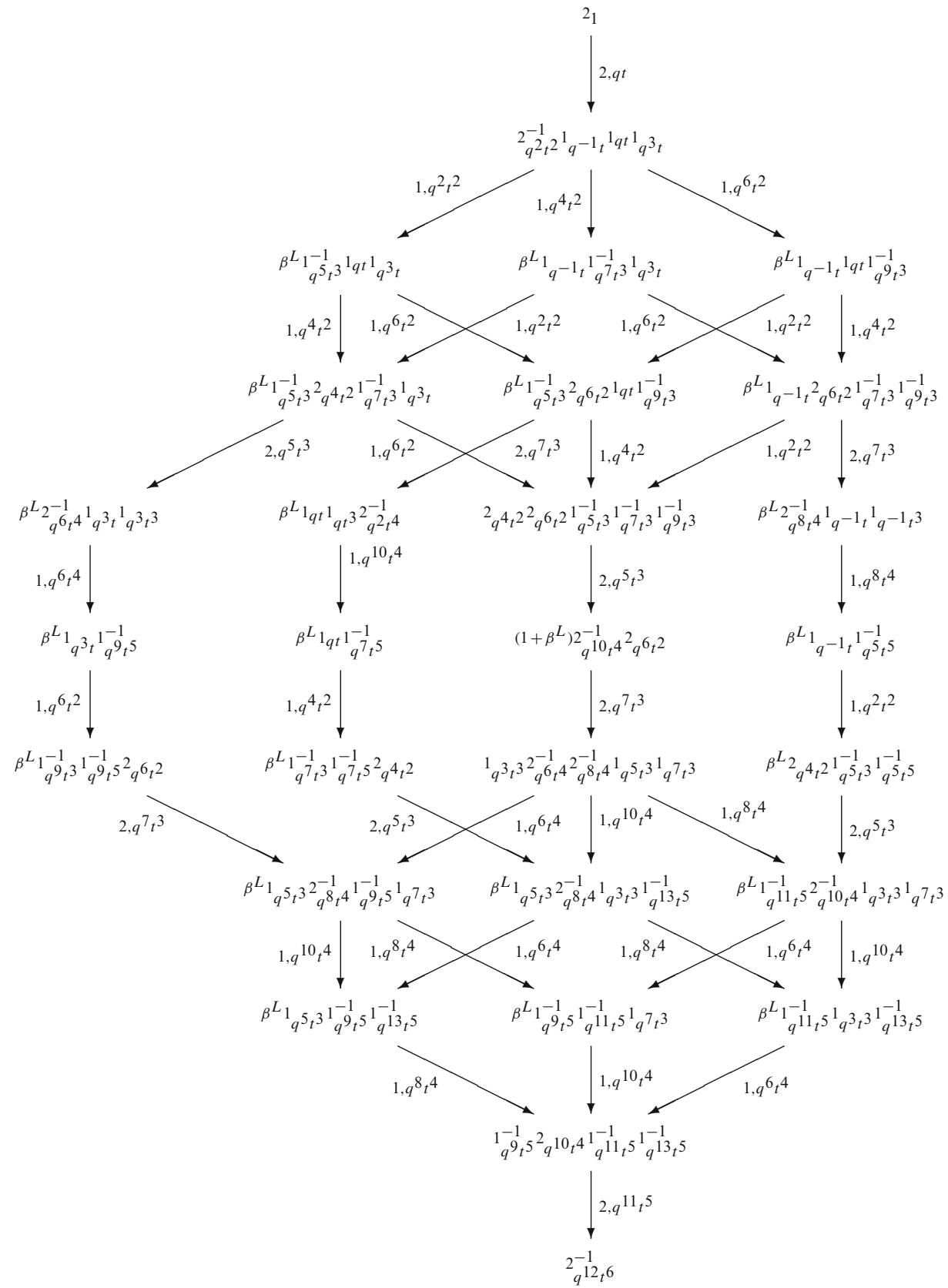

Remark 6.7. In the diagram we use the following identities which hold in $\tilde{\mathcal{Y}}_{t, q}$ : $\beta^{L} 2_{a}=\beta^{L} 2_{a q^{2}} \quad$ and $\quad \beta^{L} 1_{a}=\beta^{L} 1_{a q^{6}}$. 
It is easy to check that the expression is in the $\mathfrak{K}^{L}$. For example,

$$
\begin{aligned}
& 2_{q^{4} t^{2}} 2_{q^{6} t^{2}} 1_{q^{5} t^{3}}^{-1} 1_{q^{7} t^{3}}^{-1} 1_{q^{9} t^{3}}^{-1}+\left(1+\beta^{L}\right) 2_{q^{10} t^{4}}^{-1} 2_{q^{6} t^{2}}+1_{q^{3} t^{3}} 2_{q^{6} t^{4}}^{-1} 2_{q^{8} t^{4}}^{-1} 1_{q^{5} t^{3}} 1_{q^{7} t^{3}} \\
& =\left(\beta^{L}-1\right)+2_{q^{4} t^{2}} 2_{q^{6} t^{2}} 1_{q^{5} t^{3}}^{-1} 1_{q^{7} t^{3}}^{-1} 1_{q^{9} t^{3}}^{-1}\left(1+\widetilde{B}_{2, q^{5} t^{3}}\right)\left(1+\widetilde{B}_{2, q^{7} t^{3}}\right) \in \mathfrak{K}_{2, t, q^{L}}^{L} .
\end{aligned}
$$

The image by $\Pi_{t}^{L}$ is the twisted $t$-characters of a 29-dimensional fundamental representation of $U_{q}\left(D_{4}^{(3)}\right.$ ) (see the diagram in Section 5.2).

The image by $\Pi_{q}^{L}$ is the following $q$-character of a 7-dimensional fundamental representation of $U_{q}\left(G_{2}^{(1)}\right)$, as computed in [9, Appendix].

$$
2_{1}+2_{q^{2}}^{-1} 1_{q}+1_{q^{7}}^{-1} 2_{q^{4}} 2_{q^{6}}+2 q^{4} 2_{q^{8}}^{-1}+2_{q^{6}}^{-1} 2_{q^{8}}^{-1} 1_{q^{5}}+1_{q^{11}}^{-1} 2_{q^{10}}+2_{q^{12}}^{-1} .
$$

Now we have to consider the case of the monomial $Z_{1, \epsilon} Z_{1, \epsilon^{3}} Z_{1, \epsilon}$. The dimension of the corresponding simple module of $U_{t}\left(D_{4}^{(3)}\right)$ is $8^{3}=512$ (the module is the tensor product of three fundamental representations of dimension 8 ).

There is also an interpolating $(t, q)$-character in this case. We do not list all 512 monomials, but we list the 15 monomials which do not have $\beta^{L}$ in their coefficient:

$$
\begin{aligned}
& 1_{1} 1_{q^{2}} 1_{q^{4}} ; 1_{q^{6} t^{2}}^{-1} 1_{q^{8} t^{2}}^{-1} 1_{q^{10} t^{2}}^{-1} 2_{q^{3} t} 2_{q^{5} t} 2_{q^{7} t} ; 2 q_{q^{3} t} 2_{q^{5} t} 2_{q^{9} t^{3}}^{-1} ; 1_{q^{4} t^{2}} 1_{q^{6} t^{2}} 1_{q^{8} t^{2}} 2_{q^{3} t} 2_{q^{7} t^{3}}^{-1} 2_{q^{9} t^{3}}^{-1} ; \\
& 1_{q^{2} t^{2}} 1_{q^{4} t^{2}}^{2} 1_{q^{6} t^{2}}^{2} 1_{q^{8} t^{2}} 2_{q^{5} t^{3}}^{-1} 2_{q^{7} t^{3}}^{-1} 2_{q^{9} t^{3}}^{-1} ; 1_{q^{10} t^{4}}^{-1} q_{q^{12} t^{4}}^{-1} 1_{q^{14} t^{4}}^{-1} 2_{q^{3} t} 2_{q^{11} t^{3}} ; \\
& 1_{q^{2} t^{2}} 1_{q^{4} t^{2}} 1_{q^{6} t^{2}} 1_{q^{10} t^{4}}^{-1} 1_{q^{12} t^{4}}^{-1} 1_{q^{14} t^{4}}^{-1} q_{q^{5} t^{3}}^{-1} 2_{q^{11} t^{3}} ; 1_{q^{8} t^{4}}^{-1} 1_{q^{10} t^{4}}^{-1} 1_{q^{12} t^{4}}^{-1} 1_{q^{4} t^{2}} 1_{q^{6} t^{2}} 1_{q^{8} t^{2}} ; 2_{q^{3} t} 2_{q^{13} t^{5}}^{-1} ;
\end{aligned}
$$

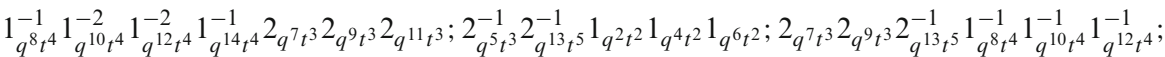

$$
\begin{aligned}
& 2_{q^{7} t^{3}} 2_{q^{11} t^{5}}^{-1} 2_{q^{13} t^{5}}^{-1} ; 2_{q^{9} t^{5}}^{-1} 2_{q^{11} t^{5}}^{-1} 2_{q^{13} t^{5}}^{-1} 1_{q^{6} t^{4}} 1_{q^{8} t^{4}} 1_{q^{10} t^{4}} ; 1_{q^{12} t^{6}}^{-1} \operatorname{1}_{q^{14} t^{6}}^{-1}{ }_{q^{16} t^{6}}^{-1} \text {. }
\end{aligned}
$$

As the other terms disappear when we specialize at $t=1$, we can compute the specialization from the above terms. We get the $q$-character of a 15-dimensional fundamental representation of $U_{q}\left(G_{2}^{(1)}\right.$ ) (image by $\Pi_{q}$ of the first example in Section 5.2).

\subsection{CONCLUSION}

We go back to the general case, that is, $r=2$ or $r=3$.

With the existence of the elementary interpolating $(t, q)$-characters in the subsections, we can conclude the proof of the main results of this section.

THEOREM 6.8. For all dominant $m$ there is a unique $F(m) \in \mathfrak{K}^{L}$ such that $m$ is the unique dominant monomial of $F(m)$.

We state its consequence in terms of KR modules.

As for $i \in I_{1}^{\vee}$, the only KR module of $U_{t}\left({ }^{L} \hat{\mathfrak{g}}\right)$ for the node $i$ with highest monomial in $\left(\mathcal{Y}_{t}^{L}\right)^{\prime}$ is trivial, we extend the definition. For $i \in I_{1}^{\vee}$, a simple $U_{t}\left({ }^{L} \widehat{\mathfrak{g}}\right)$-module with the highest monomial of the form

$$
\left(Z_{i, a} Z_{i, a t^{2}} \cdots Z_{i, a t^{2(k-1)}}\right)\left(Z_{i,-a} Z_{i,-a t^{2}} \cdots Z_{i, a t^{2(k-1)}}\right)
$$


for the double-laced case and of the form

$$
\left(Z_{1, a} Z_{1, a t^{2}} \cdots Z_{1, a t^{2(k-1)}}\right)\left(Z_{1, \epsilon^{2} a} Z_{1, \epsilon^{2} a t^{2}} \cdots Z_{1, \epsilon^{2} a t^{2(k-1)}}\right)\left(Z_{1, \epsilon^{4} a} Z_{1, \epsilon^{4} a t^{2}} \cdots Z_{1, \epsilon^{4} a t^{2(k-1)}}\right)
$$

for the triple-laced case, will also be called a KR module.

As above, we have the notion of Langlands dual representation and interpolating $(t, q)$-character $\chi \in \mathfrak{K}^{L}$ with highest monomial in $\widetilde{\mathcal{Y}}_{t, q} \backslash \alpha^{L} \widetilde{\mathcal{Y}}_{t, q}\left(\widetilde{\mathcal{Y}}_{t, q} \backslash \beta^{L} \widetilde{\mathcal{Y}}_{t, q}\right.$ in the triple-laced case): $\Pi_{t}^{L}(\chi)$ is the twisted $t$-character of a $U_{t}\left({ }^{L} \hat{\mathfrak{g}}\right)$-module and $\Pi_{q}^{L}(\chi)$ is the $q$-character of a $U_{q}(\hat{\mathfrak{g}})$-module. We obtain the following consequence of Theorem 6.8:

THEOREM 6.9. An irreducible tensor product of $K R$ modules over $U_{q}\left({ }^{L} \hat{\mathfrak{g}}\right)$ of highest monomial $M \in\left(\mathcal{Y}_{t}^{L}\right)^{\prime}$ has a Langlands dual representation. Moreover, the Langlands dual representation of a KR module over $U_{q}\left({ }^{L} \hat{\mathfrak{g}}\right)$ is a KR module over $U_{q}(\hat{\mathfrak{g}})$.

Open Access This article is distributed under the terms of the Creative Commons Attribution Noncommercial License which permits any noncommercial use, distribution, and reproduction in any medium, provided the original author(s) and source are credited.

\section{References}

1. Bouwknegt, P., Pilch, K.: On deformed $W$-algebras and quantum affine algebras. Adv. Theor. Math. Phys. 2(2), 357-397 (1998)

2. Chari, V., Hernandez, D.: Beyond Kirillov-Reshetikhin modules. Contemp. Math. 506, 49-81 (2010)

3. Chari, V., Pressley, A.: A Guide to Quantum Groups. Cambridge University Press, Cambridge (1994)

4. Chari, V., Pressley, A.: Factorization of representations of quantum affine algebras, Modular interfaces (Riverside CA 1995). AMS/IP Stud. Adv. Math., 4, 33-40 (1997)

5. Frenkel, E., Hernandez, D.: Langlands duality for representations of quantum groups. Math. Ann. (to appear)

6. Frenkel, E., Mukhin, E.: Combinatorics of $q$-characters of finite-dimensional representations of quantum affine algebras. Commun. Math. Phys. 216(1), 23-57 (2001)

7. Frenkel, E., Reshetikhin, N.: Deformations of $W$-algebras associated to simple Lie algebras. Commun. Math. Phys. 197(1), 1-32 (1998)

8. Frenkel, E., Reshetikhin, N.: The $q$-characters of representations of quantum affine algebras and deformations of $W$-algebras. Recent developments in quantum affine algebras and related topics (Raleigh, NC, 1998), pp. 163-205. Contemp. Math., vol. 248. American Mathematical Society, Providence (1999)

9. Hernandez, D.: Algebraic Approach to q,t-Characters. Adv. Math. 187(1), 1-52 (2004)

10. Hernandez, D.: The $t$-analogs of $q$-characters at roots of unity for quantum affine algebras and beyond. J. Algebra 279(2), 514-557 (2004)

11. Hernandez, D.: The Kirillov-Reshetikhin conjecture and solutions of $T$-systems. J. Reine Angew. Math. 596, 63-87 (2006)

12. Hernandez, D.: On minimal affinizations of representations of quantum groups. Commun. Math. Phys. 277, 221-259 (2007) 
13. Hernandez, D.: Kirillov-Reshetikhin conjecture: the general case. Int. Math. Res. Not. 1, 149-193 (2010)

14. Hernandez, D.: Simple tensor products. Invent. Math. 181(3), 649-675 (2010)

15. Hernandez, D., Leclerc, B.: Cluster algebras and quantum affine algebras. Duke Math. J. 154(2), 265-341 (2010)

16. Kuniba, A., Nakamura, S., Hirota, R.: Pfaffian and determinant solutions to a discretized Toda equation for $B_{r}, C_{r}$ and $D_{r}$. J. Phys. A 29(8), 1759-1766 (1996)

17. Kuniba, A., Ohta, Y., Suzuki, J.: Quantum Jacobi-Trudi and Giambelli formulae for $\mathcal{U}_{q}\left(B_{r}^{(1)}\right)$ from analytic Bethe Ansatz. J. Phys. A 28(21), 6211-6226 (1995)

18. Kuniba, A., Suzuki, S.: Analytic Bethe Ansatz for fundamental representations of Yangians. Commun. Math. Phys. 173, 225-264 (1995)

19. McGerty, K.: Langlands duality for representations and quantum groups at a root of unity. Commun. Math. Phys. 296(1), 89-109 (2010)

20. Nakajima, H.: Quiver varieties and $t$-analogs of $q$-characters of quantum affine algebras. Ann. Math. 160, 1057-1097 (2004)

21. Nakajima, H.: $t$-Analogs of $q$-characters of Kirillov-Reshetikhin modules of quantum affine algebras. Represent. Theory 7, 259-274 (2003) (electronic) 